\title{
Genome assembly, structural variants, and genetic differentiation between Lake Whitefish young species pairs (Coregonus sp.) with long and short reads
}

\section{Contributors:}

Claire Mérot ${ }^{1 *}$, Kristina S R Stenløkk ${ }^{2}$, Clare Venney ${ }^{1}$, Martin Laporte ${ }^{1,3}$, Michel Moser ${ }^{2}$, Eric Normandeau ${ }^{1}$, Mariann Árnyasi², Matthew Kent' ${ }^{2}$, Clément Rougeux ${ }^{1}$, Jullien M. Flynn ${ }^{4}$, Sigbjørn Lien², Louis Bernatchez ${ }^{1}$

*corresponding author: claire.merot@gmail.com

\section{Affiliations:}

1 Département de biologie, Institut de Biologie Intégrative et des Systèmes (IBIS), Université Laval, Québec, QC, Canada

2 Department of Animal and Aquacultural Sciences (IHA), Faculty of Life Sciences (BIOVIT), Centre for Integrative Genetics (CIGENE), Norwegian University of Life Sciences (NMBU), Ås, Norway.

3 Ministère des Forêts, de la Faune et des Parcs (MFFP) du Québec, Québec, QC, Canada

4 Department of Molecular Biology and Genetics, Cornell University, Ithaca, NY, USA

\section{Abstract}

The parallel evolution of nascent pairs of ecologically differentiated species offers an opportunity to get a better glimpse at the genetic architecture of speciation. Of particular interest is our recent ability to consider a wider range of genomic variants, not only single-nucleotide polymorphisms (SNPs), thanks to long-read sequencing technology. We can now identify structural variants (SVs) like insertions, deletions, and other structural rearrangements, allowing further insights into the genetic architecture of speciation and how different variants are involved in species differentiation. Here, we investigated genomic patterns of differentiation between sympatric species pairs (Dwarf and Normal) belonging to the Lake Whitefish (Coregonus clupeaformis) species complex. We assembled the first reference genomes for both Dwarf and Normal Lake Whitefish, annotated the transposable elements, and analysed the genome in the light of related coregonid species. Next, we used a combination of long-read and short-read sequencing to characterize SVs and genotype them at population-scale using genome-graph approaches, showing that SVs cover five times more of the genome than SNPs. We then integrated both SNPs and SVs to investigate the genetic architecture of species differentiation in two different lakes and highlighted an excess of shared outliers of differentiation. In particular, a large fraction of SVs differentiating the two species was driven by transposable elements (TEs), suggesting that TE accumulation during a period of allopatry predating secondary contact may have been a key process in the speciation of the Dwarf and Normal Whitefish. Altogether, our results suggest that SVs play an important role in speciation and that by combining second and third generation sequencing we now have the ability to integrate SVs into speciation genomics. 


\section{Introduction}

Understanding the processes underlying the evolution of species and how genomes diverge during speciation is a fundamental goal of evolutionary genomics (Jiggins, 2019; Seehausen et al., 2014). The accumulation of genomic data has allowed scientists to test evolutionary scenarios and infer the timing and circumstances of species divergence (Wolf \& Ellegren, 2017). Reciprocally, knowledge about the ecological, geographic, and demographic context of speciation helps to interpret the patterns of genetic differentiation between species (Jiggins, 2019; Ravinet et al., 2017). However, the genomewide landscape of differentiation should be interpreted with caution as it results from complex interactions between gene flow, recombination, demography, and selection (Cruickshank \& Hahn, 2014; Ravinet et al., 2017; Stevison \& McGaugh, 2020). Analysing differentiation between evolutionarily "young" pairs of species has nevertheless proven to be informative, revealing widespread heterogeneity among and between chromosomes (Henderson \& Brelsford, 2020; Martin, Davey, Salazar, \& Jiggins, 2019), sometimes identifying genes underlying reproductive isolation (Hejase et al., 2020), and informing about the number and distribution of divergent loci (Dufresnes et al., 2021). Cases of 'natural replicates', including species pairs with similar ecological and phenotypic divergence, are of particular interest, along with instances of repeated hybridization due to secondary contacts. These instances provide important insights into the genomic architecture of species differentiation (Nadeau \& Kawakami, 2019) and have revealed that similar patterns between pairs of species may be both the result of (i) shared genetic features such as low-recombination areas in which intra-specific diversity is depleted by linked selection and interspecific $F_{\mathrm{ST}}$ is inflated (Burri et al., 2015), and (ii) shared barrier loci under divergent selection or involved in reproductive isolation (Marques et al., 2016; Meier, Marques, Wagner, Excoffier, \& Seehausen, 2018).

Most of our knowledge on speciation genomics is based on single-nucleotide polymorphisms (SNPs), mainly because such variants are easily accessible with short-read sequencing (Ho, Urban, \& Mills, 2019; Mérot, Oomen, Tigano, \& Wellenreuther, 2020). However, genomes also vary in structure with loss, gain or rearrangement of sequences between individuals and between species. Such structural variants (SVs) are now recognized to be ubiquitous and to affect a larger fraction of the genomes than SNPs (Catanach et al., 2019; Feulner et al., 2013). SVs may also have a large phenotypic effect, impact recombination, and can be involved in speciation (Feulner \& De-Kayne, 2017; Kirkpatrick \& Barton, 2006; Wellenreuther \& Bernatchez, 2018). The best recognized cases are large chromosomal rearrangements such as inversions or fusions, which are hypothesized to favour speciation by preventing recombination between alternative haplotypes (Faria \& Navarro, 2010). This is supported by empirical evidence that large rearrangements can accumulate genetic incompatibilities between closely-related species of Drosophila (Noor et al., 2001) or fish (Berdan, Fuller, \& Kozak, 2021). Wholegenome duplication events are particularly prone to favour rapid diversification (Landis et al., 2018) because the rediploidization of duplicated paralogs may differ between lineages and generate hybrid incompatibilities, as observed in yeast (Scannell, Byrne, Gordon, Wong, \& Wolfe, 2006). However, small SVs may also contribute to reproductive isolation. For instance, a duplicated gene in Drosophila melanogaster leads to hybrid male sterility (Ting et al., 2004) while in crows a $2.25 \mathrm{~kb}$ transposon indel underlies plumage differences, a trait involved in mate choice between two crow species (Weissensteiner et al., 2020). More generally, the insertion, deletion, duplication and/or mis- 
regulation of transposable elements appear to be responsible for bursts of diversification and various pre- and post-zygotic barriers, particularly in plants (Serrato-Capuchina \& Matute, 2018) but also in vertebrates (Laporte et al., 2019). Overall, a better understanding of the genomic architecture of species differentiation requires the integration of structural variants into speciation genomics (Feulner \& De-Kayne, 2017; Mérot et al., 2020; Nadeau \& Kawakami, 2019). Moreover, considering both SNPs and SVs is essential to understand the cumulative effects of those different forms of genetic variation on speciation.

Two aspects of long-read sequencing, combined with the development of new bioinformatics tools, have made it possible to investigate structural variation between genomes (Ho et al., 2019; Logsdon, Vollger, \& Eichler, 2020). Firstly, long-reads have improved the contiguity and quality of genome assemblies which is particularly relevant for large and complex genomes as well as for regions riddled with repeated elements (Huddleston et al., 2014). Secondly, long-reads can be directly used to detect SVs by aligning the sequences on a reference and analysing split-reads and coverage (Mahmoud et al., 2019). Together, these have proven very powerful for making catalogues of SVs within and between species. For instance, a human genome carries on average 4,442 SVs detected by short-reads (Abel et al., 2020), and 27,662 SVs detected with long-reads (Chaisson et al., 2019). Potential restrictions when generating long-reads are the requirement for high molecular weight DNA, and potentially higher costs and lower quality. Consequently, population-level analysis of SVs via long reads is not as accessible as short read sequencing. One promising possibility is to combine technologies by performing a first step of SV discovery on a limited set of high-quality samples sequenced with long-reads, and a second step of SV genotyping on more samples sequenced with short-reads (Logsdon et al., 2020; Mérot et al., 2020).

The Lake Whitefish, C. clupeaformis, is a species complex present in numerous cold water lakes throughout North America. In particular, it comprises benthic and limnetic morphs, commonly referred to as Normal and Dwarf species, respectively, that are found in several lakes of north eastern North America which are sympatric but reproductively isolated, and as such represent a classical example of parallel speciation (Bernatchez et al., 2010; Gagnaire, Pavey, Normandeau, \& Bernatchez, 2013). Demographic modelling and the analysis of mitochondrial lineages showed that early divergence took place in allopatry during the last glaciation, and that the two glacial lineages came into secondary contact about 12,000 years ago (Bernatchez \& Dodson, 1990; Rougeux, Bernatchez, \& Gagnaire, 2017), provoking a strong character displacement in the Dwarf species toward the use of the planktonic trophic niche (Bernatchez et al., 2010; Landry, Vincent, \& Bernatchez, 2007). The Dwarf Whitefish differs in both habitat use and trophic resources, with Dwarf showing higher metabolic rates and swimming activity levels for foraging and predator avoidance relative to the Normal (Bernatchez, Chouinard, \& Lu, 1999; Rogers, Gagnon, \& Bernatchez, 2002; Trudel, Tremblay, Schetagne, \& Rasmussen, 2001). They also differ in a series of morphological, life-history, physiological, transcriptomic, and cytological traits (Dalziel, Laporte, Rougeux, Guderley, \& Bernatchez, 2017; DionCôté, Symonová, Ráb, \& Bernatchez, 2015; Martin Laporte, Dalziel, Martin, \& Bernatchez, 2016; Martin Laporte et al., 2015; Rogers \& Bernatchez, 2007; Rogers et al., 2002). The process of ecological and phenotypic divergence following secondary contact likely occurred independently, but with the same genetic background, in several post-glacial lakes (Rougeux et al., 2017). Multiple pairs of sympatric species thus provide valuable natural replicates to investigate parallelism and the genetic architecture of speciation. Moreover, as for all salmonid species (Allendorf \& Thorgaard, 1984; Lien et al., 2016; 
Macqueen \& Johnston, 2014), C. clupeaformis ancestors have undergone a past whole-genome duplication about 60MYA (Crete-Lafreniere, Weir, \& Bernatchez, 2012) followed by ongoing rediploidization, resulting in a large, complex genome of approximately $3 \mathrm{~GB}$. Therefore, structural variation between species is expected to be extensive in Lake Whitefish, though current studies have not assessed the contribution of structural variation to their speciation.

In this study, we used a combination of long-read and short-read sequencing (Fig. 1) to investigate the genetic architecture of speciation and address the contribution of SVs to the genomic differentiation of Dwarf and Normal Whitefish. The main goal was to provide high-quality genomic resources for $C$. clupeaformis in order to investigate parallel and non-parallel genomic patterns of differentiation between Dwarf and Normal species in two independent North American lakes. We started by assembling the first reference genome of the Normal species (C. clupeaformis cf. Normal) based on one sample sequenced with long-reads and a genetic map. We documented the specificities of the genome to explore the remaining traces of previous whole-genome duplication and annotated the whitefish transposable elements. Next, we added a second reference assembly of a Dwarf individual based on long-reads, and used a combination of assembly comparison, long-reads, and short-reads to characterize SVs between and within Dwarf and Normal species. Finally, we analysed genome-wide landscapes of differentiation at the population level using 32 samples (17 Dwarf and 15 Normal Whitefish) from two different lakes sequenced with short-reads. We tested the hypothesis that the two lakes would show parallel patterns of differentiation between Dwarf and Normal Whitefish and compared signals observed with different kinds of variants (SNPs vs. SVs). Our study provides a unique opportunity to characterize the contribution of both SNPs and SVs to differentiation between young species pairs, with important implications for our understanding of speciation in general.

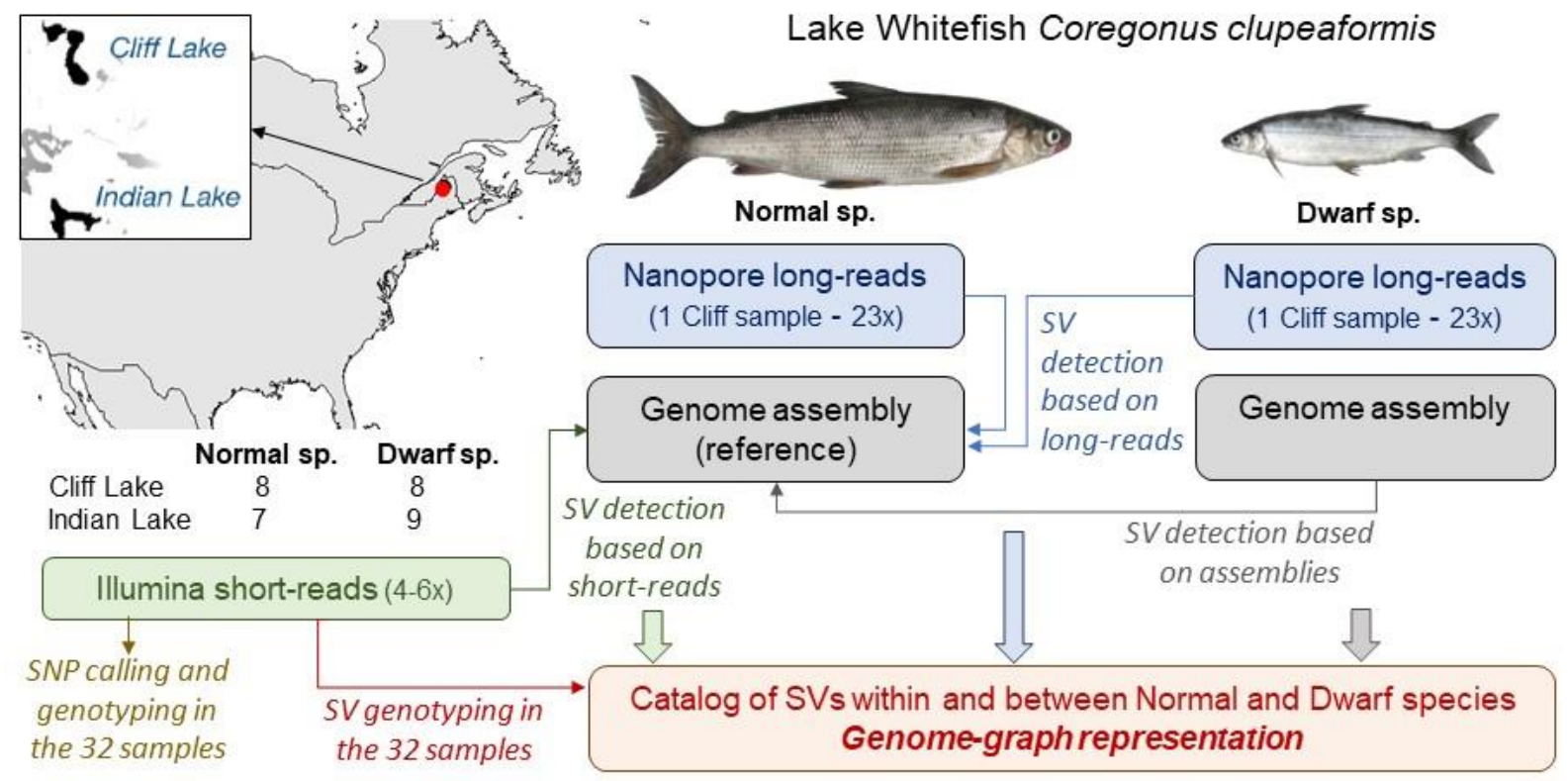

\section{Figure 1: Study design}

Overview of the sampling and sequencing design, which included 32 wild samples of $C$. clupeaformis Normal sp. and Dwarf sp. from Cliff Lake and Indian Lake in Maine (USA), sequenced by Illumina short reads, as well as two samples from Cliff Lake (one Normal and one Dwarf), sequenced by Nanopore long reads to assemble genomes. Schematic representation of the subsequent analysis performed to detect and genotype structural variants (SVs) with different datasets. 
bioRxiv preprint doi: https://doi.org/10.1101/2022.01.15.476463; this version posted January 16,2022 . The copyright holder for this preprint (which was not certified by peer review) is the author/funder, who has granted bioRxiv a license to display the preprint in perpetuity. It is made available under aCC-BY-NC-ND 4.0 International license.

\section{Methods}

\section{Sampling, DNA extraction and sequencing of Coregonus clupeaformis}

\section{- Long-read sequencing}

For long-read sequencing and the assembly of both reference genomes, we sampled one adult from the Normal species and one adult from the Dwarf species. Dwarf and Normal Lake Whitefish were sampled from Cliff Lake, Maine (46.3991,-69.2491) in June 2010. Fish were caught live with gillnets, euthanized, immediately dissected to obtain fresh tissue samples, and sexed following a protocol described previously in Evans \& Bernatchez (2012). Muscle samples were flash frozen in liquid nitrogen and later stored at $-80^{\circ} \mathrm{C}$. High molecular weight DNA was extracted from $40 \mathrm{mg}$ frozen liver from both species using Qiagen Genomic Tip 100/G kit (Qiagen, Hilden, Germany). DNA integrity was assessed visually by separating fragments on a $0.5 \%$ TAE agarose gel which revealed a predominant band of high molecular weight DNA $>45 \mathrm{~kb}$. Smaller fragments were removed by performing size selection, with >20kb cutoff, using a High Pass Plus cassette (BPLUS10) run on a Blue Pippin (Sage Scientific, MA, USA). Using 1.6ug of size selected DNA, four sequencing libraries were independently generated for each samples using the SQK-LSK109 sequencing kit (Oxford Nanopore Technologies, Oxford, UK), according to the "Genomic DNA by Ligation Nanopore" protocol. For each species, multiple PromethION flow cells (VR9.4.1; ONT) were loaded with library. Run performance was monitored, and once the number of sequencing pores dropped below $10 \%$ the starting number, the run was stopped and a nuclease flush was performed using the NFL_9076_v109_revA Nuclease Flush protocol from Oxford Nanopore. Additional library material was loaded onto flow-cells (by species) and sequencing initiated. In total, 3 FC were used to sequence the Dwarf sample (with 3 reloads among them) and 3 FC for the Normal sample (with 3 reloads). Raw nanopore reads were base-called using Guppy (v3.0.5. flip-flop HAC model). Data metrics before quality filtering were $72.1 \mathrm{~Gb}(\mathrm{~N} 50=27.1 \mathrm{~Kb})$ for the Dwarf sample and $80 \mathrm{~Gb}(\mathrm{~N} 50=27.9 \mathrm{~Kb})$ for the Normal sample.

\section{- Short-read sequencing}

For population-level analysis, we sampled and sequenced with Illumina short reads, 32 C. clupeaformis including 8 Normal and 8 Dwarf from Cliff Lake, Maine (46.3991,-69.2491), and 7 Normal and 9 Dwarf from Indian Lake, Maine (46.2574, -69.2987). Fish were caught live with gillnets, euthanized and immediately dissected to obtain fresh tissue samples. Samples were stored in RNAlater and DNA was extracted using a modified version of a salt extraction protocol (Aljanabi \& Martinez, 1997). Libraries were prepared and samples were sequenced aiming for 5-6X coverage on a HiSeq2500 instrument at the McGill Genome Québec Innovation center (Montréal, CQC, Canada).

Paired short reads were trimmed and filtered for quality with FastP (Chen, Zhou, Chen, \& Gu, 2018), aligned to the reference genome of the Normal C. clupeaformis (see below) with BWA-MEM (Li \& Durbin, 2009), and filtered to keep mapping quality over 10 with Samtools v1.8 (Li et al., 2009). Duplicate reads were removed with MarkDuplicates (PicardTools v1.119.) We realigned around indels with GATK IndelRealigner (McKenna et al., 2010) and soft clipped overlapping read ends using clipOverlap in bamUtil v1.0.14 (Breese \& Liu, 2013). The pipeline is available at https://github.com/enormandeau/wgs sample preparation 
bioRxiv preprint doi: https://doi.org/10.1101/2022.01.15.476463; this version posted January 16, 2022. The copyright holder for this preprint (which was not certified by peer review) is the author/funder, who has granted bioRxiv a license to display the preprint in perpetuity. It is made available under aCC-BY-NC-ND 4.0 International license.

Assembly and annotation of two reference genomes for Coregonus clupeaformis

\section{- De novo assembly and polishing}

Long reads were filtered for a minimum length of 4000 bps and minimum average quality PHRED score of 7.This resulted in a total of $62.9 \mathrm{~Gb}(\mathrm{~N} 50=28.5 \mathrm{~kb}, \mathrm{~N} 90=16.3 \mathrm{~kb})$ for the Normal and $60.8 \mathrm{~Gb}$ ( $\mathrm{N} 50=27.4 \mathrm{~kb}, \mathrm{~N} 90=15.0 \mathrm{~kb}$ ) for the Dwarf. For the Normal assembly, filtered long reads were independently assembled using Flye (Kolmogorov, Yuan, Lin, \& Pevzner, 2019) (version 2.5, default parameters) three times using overlap sizes of $8 \mathrm{~kb}, 10 \mathrm{~kb}$ and $15 \mathrm{~kb}$ (Table S1) . The three resulting assemblies were merged into a final assembly with Quickmerge (Chakraborty, Baldwin-Brown, Long, \& Emerson, 2016) (v0.3, options: -hco 5.0 -c 1.5 -I 2000000 -ml 10000). For the Dwarf assembly, filtered long reads were assembled using Flye (version 2.5, default parameters) using overlap sizes of $10 \mathrm{~kb}$. The final assemblies were first polished with their respective long reads using marginPolish (v1.2.0 https://github.com/UCSC-nanopore-cgl/MarginPolish) for the Normal and PEPPERPolish (default settings, model: pepper_r941_guppy305_human.pkl.) for the Dwarf. In a second step, each assembly was polished with short reads using Pilon (Walker et al., 2014) requiring a minimal coverage of $3 x$ to polish (v1.23, --mindepth 3). BUSCO (Benchmarking Universal Single-Copy Orthologs) scores were computed to assess gene space completeness by looking for the presence or absence of highly conserved genes (BUSCO v3.0.2, reference database: actinopterygii_odb9 -sp zebrafish). BUSCO scores for the Flye polished assemblies were $\mathrm{C}: 94.4 \%[\mathrm{~S}: 50.9 \%, \mathrm{D}: 43.5 \%], \mathrm{F}: 1.7 \%$, $\mathrm{M}: 3.9 \%, \mathrm{n}: 4584$ for the Normal and C:94.6\%[S:59.1\%,D:35.5\%], F:0.9\%, M:4.5\%, n:4584 for the Dwarf. Out of 4584 searched BUSCO gene groups about $94 \%$ were detected as singletons (S) or duplicates $(D)$, a small fraction were missing $(M)$ or fragmented $(F)$.

\section{- Scaffolding into chromosomes with a genetic map}

To anchor the contigs into chromosomes, we built a linkage map from previously-published data, including 100 full-sibs and their two parents sequenced with reduced-representation sequencing (Gagnaire, Normandeau, Pavey, \& Bernatchez, 2013). Genotype likelihoods were obtained with SAMtools mpileup ( $\mathrm{Li}$ et al., 2009) following the pipeline and parameters provided in lep-map3 documentation (Rastas, 2017). Only positions with at least $3 X$ coverage were kept. Linkage map was built from progeny and parents of a backcross family (mother: Dwarf $x$ Normal hybrid; father: pure Dwarf) details in Rogers, Isabel, \& Bernatchez (2007)) using Lep-MAP3 (Rastas, 2017) following a pipeline available at https://github.com/clairemerot/lepmap3 pipeline. With the Filtering module, markers with more than $50 \%$ of missing data, that were non-informative, and with extreme segregation distortion $\left(\chi^{2}\right.$ test, $P<10^{-12}$ ) were excluded. Markers were assigned to linkage groups (LGs) using the SeparateChromosomes module with increasing values of the logarithm of the odds (LOD) from 8 to 11 and a minimum size of 20 markers. Markers unassigned to LGs, or released from LG correction, were subsequently joined to LGs using the module JoinSingle with decreasing values of LOD until LOD $=3$ and a minimum LOD difference of 1 . This procedure assigned 5,188 markers into 40 LGs. Within each LG, markers were ordered with 10 iterations of the OrderMarker module. The marker order from the run with the best likelihood was retained and refined 10 times with the evaluateOrder flag with 5 iterations each. To account for the lower recombination rate in male salmonids compared to females, we adjusted the parameter of recombination rates accordingly (recombination1 $=0.0005$; recombination2 $=0.0025$ ). Exploration for more stringent filtering for missing data, different values of LOD or by keeping only female-informative markers resulted in very consistent and collinear maps but with less markers, whose density is critical to accurately scaffold the genome. 
Since Dwarf and Normal Whitefish have the same number of chromosomes (Dion-Côté et al., 2015) and the genetic map was built from a backcross family, we used the same map to improve both the Dwarf and the Normal genome assemblies. Scaffolds were assembled into chromosomes using Chromonomer (Catchen, Amores, \& Bassham, 2020), which anchors and orients scaffolds based on the order of markers in the linkage map. The default parameters were used. In both assemblies, chromosomes were renamed to match homologous chromosomes in the reference genome of the European sister species, Coregonus sp. "Balchen" (De-Kayne, Zoller, \& Feulner, 2020), as detailled in Table S2. For all subsequent analyses, the Normal Whitefish genome was chosen as the reference.

\section{- Annotation for genes and transposable elements}

Gene content annotation of the Normal and the Dwarf Whitefish genomes was made with the NCBI Prokaryotic Genome Annotation Pipeline using the following transcriptome sources available on NCBI: Dion-Côté: PRJNA237376; Rougeux: 72 liver RNA samples from 2018, NCBI: PRJNA448004; Carruthers: SRR6321817, SRR6321818, SRR6321819, SRR6321820, SRR6321821, SRR6321822, SRR6321823, SRR6321824; Pasquier: SRP058861 Lake Whitefish, SRP045143 European Whitefish.

We used RepeatModeler2 (Flynn et al., 2020) to annotate transposable elements in our assembly. We had to slightly modify the RepeatModeler LTR pipeline because of an incompatibility between this genome and the LTRHarvest dependency. We instead substituted LTRfinder-parallel (Ou \& Jiang, 2019) to identify LTRs in the genome. We combined the LTR-specific library with the general repeat library as done in canonical RepeatModeler2. After obtaining the TE library, we re-labelledthe fasta headers of sequences that were identified in the LTR pipeline but were assigned an "Unknown" classification due to lack of homology to database sequences, to broadly classify them as LTR elements. We then used RepeatMasker to annotate the locations of each repeat family in the genome. We used parseRM.pl (https://github.com/4ureliek/Parsing-RepeatMasker-Outputs/blob/master/parseRM.pl) to summarize the genomic abundance of each TE subclass (LTR, LINE, SINE, DNA-TIR, Helitron), correcting for overlapping masking which sometimes occurs with RepeatMasker. We also used parseRM.pl to produce a landscape plot of the genome composition, where the TE-subclass composition is shown in one percent divergence windows (compared to each TE copy's respective consensus sequence), where low divergence sequences suggest more recent insertions and higher divergence sequences suggest older insertions.

\section{- Synteny, map, chromosomes, and genome analysis}

To analyse synteny with related species, we first compared the linkage map to the previously-published maps of C. clupeaformis (Gagnaire, Normandeau, et al., 2013), C. lavaretus "Albock" (De-Kayne \& Feulner, 2018) and C. artedii (Blumstein et al., 2020) using MapComp (Sutherland et al., 2016), a program designed to compare syntenic relationships among markers between linkage maps of any related species using an intermediate genome, here, our reference genome. Correspondence between chromosomes and linkage groups across maps of different Coregonus $s p$. is provided in Table S2 and Fig S1-S3.

Next, we aligned the repeat-masked Normal Whitefish reference genome to the European Whitefish reference, Coregonus lavaretus "Balchen" (De-Kayne et al., 2020) with nucmer (-I 100 -c 500, (Marçais et al., 2018)) and used Symap v4.2 (Soderlund, Bomhoff, \& Nelson, 2011) to extract syntenic blocks 
along the genome. Syntenic blocks were visualised in R using the package Circlize (Gu, Gu, Eils, Schlesner, \& Brors, 2014).

To investigate chromosome types (acrocentric, metacentric), we used phased information from the linkage map by applying a method developed by (Limborg, McKinney, Seeb, \& Seeb, 2016), which uses phased progeny genotypes to detect individual recombination events. The cumulative number of recombination events between the first marker and increasingly distant markers was computed from both extremities of each chromosome and this recombination frequency (RFm) is expected to reach a plateau over a chromosome arm (see Limborg et al. (2016) for details and Fig. S4).

As salmonids have experienced an ancestral whole-genome duplication, most of the chromosomes are expected to be homologous to another one, and some pairs still recombine to a certain extent, resulting in pseudo-tetrasomal regions or chromosomes (Glasauer \& Neuhauss, 2014; Lien et al., 2016; Sutherland et al., 2016). To investigate this homology, we explored self-synteny by aligning the repeatmasked Normal Whitefish genome on itself with nucmer (-maxmatch -I 100 -c 500, (Marçais et al., 2018) ) and extracted syntenic blocks with Symap v4.2 (Soderlund et al., 2011). The degree of sequence similarity within each of the syntenic blocks was calculated after a subsequent alignment with Lastz (Harris, 2007), following the procedure described in Lien et al. (2016). To assign Lake Whitefish chromosomes to ancestral chromosomes following the nomenclature proposed by Sutherland et al. (2016) based on Northern Pike (Esox Lucius) linkage groups, we aligned the repeat-masked Normal genome to the Northern Pike reference genome with Minimap2 (Li, 2018) and visualise alignment using D-Genies (Cabanettes \& Klopp, 2018).

We further explored whether the assembly included duplicated or collapsed regions by quantifying variation of coverage along the genome. Total depth of aligned short reads across the 32 samples was calculated using ANGSD (Korneliussen, Albrechtsen, \& Nielsen, 2014) at each position with the option -doDepth -dumpCounts, and averaged by sliding-windows of $100 \mathrm{~kb}$. The coordinates of putativelycollapsed regions is provided in Table S3.

\section{Detection and characterisation of structural variants}

- SV detection based on the comparison of de novo assemblies

Structural variants between the Normal and the Dwarf Whitefish haploid assemblies were identified using three independent approaches detailed below. All methods included an alignment step between the Normal Whitefish reference and the Dwarf Whitefish assembly. To avoid artefacts due to scaffolding with a map, we chose to use the contig-level assembly for the query genome (i.e. the Dwarf Whitefish).

(i) We built a genome-graph with the two assemblies using Minigraph (Li, Feng, \& Chu, 2020) with the -xggs optionsand retrieved SVs in a bed format with gfatools-bubble. The graph with variants was further reformatted into a vcf with full sequence information using vg suite (Hickey et al., 2020) and custom scripts.

(ii) We aligned the assemblies with Minimap2 (Li, 2018) and parameters -a -x asm5 --cs -r2k, and extracted SVs with Svim-Asm (Heller \& Vingron, 2020) and the following parameters: --haploid --min_sv_size $50 \quad$--max_sv_size $200000 \quad$--tandem_duplications_as_insertions -interspersed_duplications_as_insertions. 
(iii) We ordered the scaffolds of the Dwarf Whitefish assembly according to the Normal Whitefish reference using Ragtag (Alonge et al., 2019) and aligned the assemblies with Minimap2 (Li, 2018) and parameters "-ax asm5" and ran SyRI (Goel, Sun, Jiao, \& Schneeberger, 2019) with standard parameters.

The SVs detected by the three methods were filtered to remove translocations and breakends, short SVs below 50bp, and SVs which overlapped a scaffold junction (characterized by a gap of $10 \mathrm{~N}$ ). The three VCFs were joined using Jasmine (Kirsche et al., 2021) and the following parameters "-ignore_strand --mutual_distance --max_dist_linear $=0.5$--min_dist $=100$ ", and we kept SVs detected by at least 2 approaches. All scripts are available at https://github.com/clairemerot/assembly SV

\section{- SV detection based on long reads}

We mapped long reads to the reference using Winnowmap2 v2.0 with the "--MD" flag to better resolve repetitive regions of the genome (Jain et al., 2020). Sam-files were sorted and converted into BAMfiles with samtools V1.3.1 ( $\mathrm{Li}$ et al., 2009). The SV-detection was performed with three long-read specific SV calling programs: Sniffles v1.0.12 (Sedlazeck et al., 2018) (-I 50 -s 7 -n -1), SVIM v1.2.0 (Heller \& Vingron, 2019) (--insertion_sequences) and NanoVar v1.3.9 (Tham et al., 2020) with default settings. SVs of type breakpoint were removed, and other excess information in the VCF files were filtered out using custom R scripts. In addition, read names were added to preserve insertion sequences in the final VCF. We kept SVs detected by at least two callers after merging with Jasmine v1.1.0 (Kirsche et al., 2021) including refinement of insertion sequences with Iris "max_dist_linear=0.1 min_dist=50 -default_zero_genotype --mutual_distance min_support=2 --output_genotypes --normalize_type -run_iris iris_args=--keep_long_variants". Custom $R$ scripts available at https://github.com/kristinastenlokk/long read SV

\section{- SV detection based on short reads}

Structural variants among the 32 samples sequenced with short reads were identified using three independent approaches: (i) Manta ( Chen et al., 2016), (ii) the Smoove pipeline (https://github.com/brentp/smoove) which is based on Lumpy (Layer, Chiang, Quinlan, \& Hall, 2014) and (iii) Delly (Rausch et al., 2012). All of the approaches rely on the filtered bam files resulting from the alignment of the short reads to the Normal Whitefish reference (as described above). All SV callers were run with defaults parameters except for Smoove that was run by subsets of chromosomes, and Delly by subsets of individuals. VCF outputs were formatted with custom scripts to include full sequence information and filtered to remove translocations, breakends, short SVs below 50bp, and SVs which overlapped a scaffold junction in the reference (characterized by a gap of $10 \mathrm{~N}$ ). The three VCFs were joined using Jasmine (Kirsche et al., 2021) and the following parameters "--ignore_strand -mutual_distance --max_dist_linear=0.5 --min_dist=50 --max_dist=5000 --allow_intrasample", and we kept SVs detected by at least 2 approaches. All scripts are available at https://github.com/clairemerot/SR SV

\section{- Analysis and annotation of SVs}

Structural variants detected by the three kinds of datasets (assembly comparison, long reads, short reads) were joined using Jasmine (Kirsche et al., 2021) and the following parameters --ignore_strand -mutual_distance --max_dist_linear=0.5 --min_dist=100 --min_overlap 0.5". We explored the overlap of SVs detected in more than one dataset according to its type (deletion: DEL, insertion: INS, inversion: INV, duplication: DUP) and its size. The sequences included in SVs (e.g. the reference sequence in the 
case of a deletion, or the alternative sequence in the case of an insertion) were annotated for transposable elements using RepeatMasker and the TE library of the Normal C. clupeaformis (see above). We explored the length of SV sequences covered by TE or simple repeats quantitatively (Table S4-S5) and also categorized them as associated with TE or other kinds of repeats if more than $50 \%$ of the SV sequence was covered by a given TE family or other kind of repeats.

\section{Analysis of single-nucleotide and structural polymorphisms}

\section{- SNPs calling and genotyping}

To detect SNPs and genotype them, we analysed the short reads of the 32 samples, in bam format, with the program ANGSD v0.931 (Korneliussen et al., 2014), which accounts for genotype uncertainty and is appropriate for medium coverage whole genome sequencing (Lou, Jacobs, Wilder, \& Therkildsen, 2020). Input reads were filtered to remove low-quality reads and to keep mapping quality above 30 and base quality above 20. Genotype likelihoods were estimated with the GATK method (-GL 2). The major allele was the most frequent allele (-doMajorMinor 1). We filtered to keep positions covered by at least one read in at least $50 \%$ of individuals, with a total coverage below 800 ( 25 times the number of individuals) to avoid including repeated regions in the analysis. From this list of variant and invariant positions, we selected SNPs with a minor allele frequency (MAF) above $5 \%$ and subsequently used this SNP list with their respective major and minor alleles for most analyses, including PCA, $F_{S T}$, and allelic frequency difference (AFD).

\section{- SVs genotyping}

To genotype the identified SVs in the 32 samples, we used a genome-graph approach with the suite of tools vg (Garrison et al., 2018; Hickey et al., 2020). Briefly, the full catalog of SVs discovered through assembly comparison and long reads and short reads SV calling was combined with the reference genome to build a variant-aware graph using the module $v g$ autoindex -giraffe. Short reads from the 32 samples were then aligned to the graph with the module vg giraffe (Sirén et al., 2020). For each SV represented in the graph through a reference and an alternative path, a genotype likelihood was calculated with the module $v g$ call. We then combined the VCFs of SV genotype likelihoods across the 32 samples. For population-level analysis, mirroring the filters applied for SNPs, we retained SVs covered by at least one read in at least $50 \%$ of the samples, and with an alternative allele frequency between $5 \%$ and $95 \%$. The pipeline used is available here https://github.com/clairemerot/genotyping SV. Subsequent analytical steps were performed in ANGSD, which allows population-level analysis within a genotype likelihood framework, using the VCF of SV genotype likelihoods as input.

\section{- Genetic differentiation according to lake and species}

An individual covariance matrix was extracted from the genotype likelihoods of SNPs and SVs in beagle format using PCAngsd (Meisner \& Albrechtsen, 2018). The matrix was decomposed into PCs with R using a scaling 2 transformation which adds an eigenvalue correction (Legendre \& Legendre, 2012). Pairwise $F_{S T}$ differentiation between all populations was estimated based on the allele frequency spectrum per population (-doSaf) and using the realSFS function in ANGSD. Minor allelic frequencies per population (MAF) were estimated based on genotype likelihoods using the function doMaf in ANGSD. We then computed allelic frequency difference (AFD) between sympatric species in each lake for each variant as AFD=maf(Dwarf) - maf(Normal). AFD is a polarised difference of 
bioRxiv preprint doi: https://doi.org/10.1101/2022.01.15.476463; this version posted January 16, 2022. The copyright holder for this preprint (which was not certified by peer review) is the author/funder, who has granted bioRxiv a license to display the preprint in perpetuity. It is made available under aCC-BY-NC-ND 4.0 International license.

frequency that varies between -1 and 1 , meaning that when we compared AFD between lakes they can be either with same sign (the same allele has a higher frequency in the same species in both lake) or opposite sign (the allele more frequent in the Dwarf in one lake is more frequent in the Normal in the other lake). For $\mathrm{F}_{S T}$ and AFD estimates, positions were restricted to the polymorphic SNPS/SVs (>5\% MAF) previously assigned as major or minor allele (options -sites and-doMajorMinor 3), and which were covered in at least $50 \%$ of the samples in each population. Given the high density of SNPs, F $_{\text {ST }}$ and mean absolute AFD were also calculated by windows of $100 \mathrm{~kb}$ for visualisation. The most differentiated variants between species were defined in each lake as the ones within the upper $95 \%$ quantile for $\mathrm{F}_{\mathrm{ST}}$, and in below the $2.5 \%$ or above the $97.5 \%$ quantile for AFD. By chance only, we would expect that $0.5 \%$ of variants are in the upper $F_{S T}$ quantile in both lakes, $0.25 \%$ of variants are in AFD outliers in both lakes with same sign, and $0.25 \%$ of variants are in AFD outliers in both lakes with opposite sign. We used Fisher's exact test to determine whether the number of outlier variants overlapping between lakes exceeded this expectation.

Using BEDtools, we extracted the list of genes overlapping with the most differentiated SNPs/SVs. We then tested for the presence of overrepresented GO terms using GOAtools (v0.6.1, $P_{\text {val }}=0.05$ ) and filtered the outputs of GOAtools to keep only GO terms for biological processes of levels 3 or more, and with an FDR value equal to or below 0.1. Using our annotation of TEs and repeated sequences on SVs, we tested for overrepresentation of SVs associated with particular families of TEs within outliers using a Fisher test.

Finally, several QTL for behavioural, morphological and life-history traits differentiating Normal and Dwarf Whitefish previously identified in Gagnaire, Normandeau, et al.(2013) and Rogers et al. (2007) were positioned on the Normal Whitefish reference genome. We compared the positions of those QTL relative to the most differentiated regions and extracted the list of genes hit by an outlier variants and belonging to a $1 \mathrm{Mb}$ window around the QTL.

\section{Results:}

\section{High-quality reference assembly for Coregonus clupeaformis Normal species}

Using long-read sequencing, we built the first reference genome assembly for the Normal Whitefish species of Coregonus clupeaformis (ASM1839867v1). The de novo assembly showed good contiguity with a N50 of $6.1 \mathrm{Mb}$ and a L50 of 101 contigs. A linkage map allowed us to anchor and orient $83 \%$ of the genome into 40 linkage groups, the expected number of chromosomes for Lake Whitefish (DionCôté et al., 2015; Phillips, Reed, \& Ráb, 1996). Studying recombination along those linkage groups, we identified 7 metacentric chromosomes, 3 putatively metacentric (or sub-metacentric chromosomes) and 30 acrocentric chromosomes (Fig. S4, Fig. 2A). The final assembly included 40 chromosomes and 6427 unanchored scaffolds with a N50 of $57 \mathrm{Mb}$ for a total genome size of $2.68 \mathrm{~Gb}$. This reference genome had a high level of completeness, with 94\% (actinopterygii) of universal single-copy orthologous genes. A relatively high percentage of duplicated BUSCO groups (43\%) was observed, which is a consequence of the salmonid-specific whole genome duplication.

The genome was composed of $60.5 \%$ of transposable elements (Figure S5, Table S4). The greatest transposable element subclass representation in terms of total base pairs was DNA-TIR elements, taking up $24 \%$ of the genome. LINEs and LTRs were approximately equally abundant at about $13 \%$ of the genome each. Elements that were unclassified took up 9\% of the genome. SINEs took up less than 
$1 \%$ of the genome, and rolling-circle/helitron elements were essentially absent. Our repeat identification pipeline identified 3490 distinct families. LTR elements were the most diverse with 1521 families identified, almost half the total number of families. Comparatively, 373 families were identified as DNA-TIR elements and 250 as LINEs. The genome is composed of TEs at a variety of stages of decomposition (Figure S6). A proxy for age of a given insertion is its sequence divergence from the consensus sequence, since the longer the insertions have been present, the more time there has been for accumulation of random mutations. The landscape plot shows that an equal amount (in terms of bp) of LINEs, LTRs, and DNA-TIRs are present in recent insertions less than $1 \%$ diverged from the consensus sequence. DNA-TIR elements near the $5 \%$ divergence level are the most abundant, indicating an older burst of activity.

The $C$. clupeaformis genome showed high synteny with the closely-related European Alpine whitefish, Coregonus lavaretus "Balchen" (Fig. 2A), allowing the identification of 39 homologous chromosomes which were named accordingly. Chromosome 40 of Coregonus lavaretus "Balchen" was small and had no homologous chromosome in the $C$. clupeaformis genome. Chromosome 40 of $C$. clupeaformis aligned with a fraction of chromosome 4 in the "Balchen" assembly and may or may not be one arm of the putatively-metacentric Chromosome 4. Some chromosomes (Chr7, Chr8, Chr15, Chr17, Chr20, Chr28, Chr35) included syntenic blocks matching two chromosomes in the related species. Some of those blocks likely correspond to duplicated regions collapsed in one of the assemblies, as they also exhibit higher than average coverage. Those blocks may also belong to pseudo-tetrasomal chromosomes, which are homeologous chromosomes resulting from ancient whole-genome duplication and that still recombine to a certain extent (Allendorf et al., 2015; Blumstein et al., 2020; Lien et al., 2016; Waples, Seeb, \& Seeb, 2016).

The identification of ancestral chromosomes by alignment to other linkage maps (Fig. S1-S3) and to the Northern Pike genome (Fig. S7), as well as self-synteny (Fig. 2A), allowed us to further identify the pairs of homeologous chromosomes. A few regions (Chr22, Chr 32, the end of Chr1) show no matching region in the $C$. clupeaformis genome but high coverage, suggesting that the assembly may have locally collapsed two highly similar regions (Fig. S2A, Table S3). Self-synteny assessment also supports fusion events between ancestral chromosomes that were previously reported in the three Coregonus species, C. lavaretus, C. artedii and C. clupeaformis (Blumstein et al., 2020; Sutherland et al., 2016) such as PK05-PK06 (Chr01), PK10-PK24 (chr8), PK11-PK21 (Chr7), PK01-PK14 (Chr15), PK16-PK23 (Chr4), PK8PK9 (Chr20), as well as putative complex rearrangements between PK10-PK20-PK23 (Chr17, Chr28, Chr4). Those 8 chromosomes also correspond to the ones identified as metacentric in our study and in the Cisco C. artedii (Blumstein et al., 2020). 

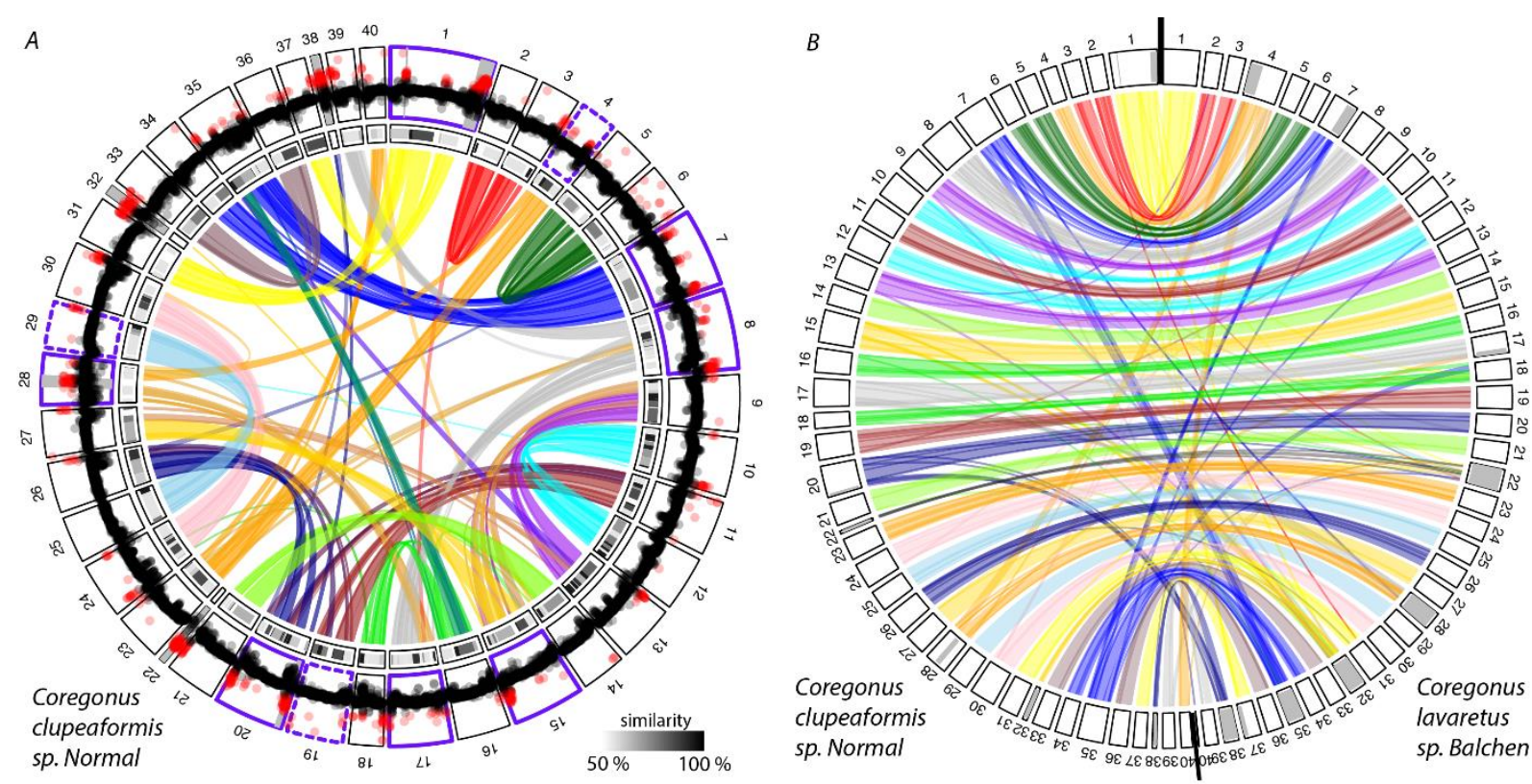

Figure 2: Self-synteny and coverage in C. clupeaformis sp. Normal, and synteny with C. lavaretus Balchen,

(A) Circular plot showing syntenic relationship between homoeologous Lake Whitefish chromosomes (inner track) and their level of sequence similarity (medium track). The outer track display mean coverage by windows of $100 \mathrm{~kb}$ in the short reads alignments. Points coloured in red show coverage higher than 1.5 times the average coverage (3.7X). Chromosomes surrounded by a purple outline are metacentric chromosomes, with dashed lines for putatively metacentric chromosomes. (B) Circular plot showing syntenic relationship between C. clupeaformis sp. Normal and C. lavaretus Balchen. On both plots, chromosomes are coloured according to the ancestral origin (based on the PK nomenclature proposed in Sutherland et al (2016). Regions coloured in grey represent collapsed duplicated regions in genome assemblies.

\section{Discovery of structural variation using a combination of sequencing tools}

To identify structural variants between Normal and Dwarf Whitefish, we built a de novo assembly of a Dwarf Whitefish individual (ASM2061545v1) by combining both long-read and short-read sequences. This assembly shows high contiguity with a N50 of $2.2 \mathrm{Mb}$ and a L50 of 274 contigs, of which $73 \%$ could be placed into chromosomes using the linkage map. The final Dwarf assembly included 40 chromosomes and 7294 unanchored scaffolds with a N50 of $52 \mathrm{Mb}$ for a total genome size of $2.76 \mathrm{~Gb}$. The comparison of the Dwarf assembly to the Normal reference unveiled a total of 244,717 SVs, of which 89,909 were detected by at least two tools and were kept as "high-confidence SVs". Approximately half of the SVs were deletions and half were insertions (Fig. 3A). Duplications were counted as insertions, and only a limited number of inversions were detected $(2,815$, out of which only 77 were found by two tools).

Since a comparison of haploid assemblies is only able to detect SVs in the Dwarf relative to the Normal, and may be sensitive to assembly errors, we next investigated SV polymorphisms based on long reads. This unveiled a higher number of high-confidence SVs with a total of 194,861 SVs detected by at least two tools. Those included SVs putatively heterozygous in the Normal and the Dwarf genomes and resulted in a high number of novel deletions and insertions.

Only two samples (one Dwarf and one Normal) were sequenced with long reads; hence we hoped to cover a wider range of population structural polymorphism by using short reads on 32 individuals to 
detect SVs. This method nevertheless appeared less powerful as 84,673 SVs were detected, with only 28,579 detected by at least two tools. The large majority of SVs detected in this dataset were deletions ( $n=77,899 ; 92 \%)$, followed by duplications $(n=5,927 ; 7 \%)$, a few inversions $(n=24 ; 0.02 \%)$ and insertions $(n=15 ; 0.01 \%)$ (Fig. 3A).

A

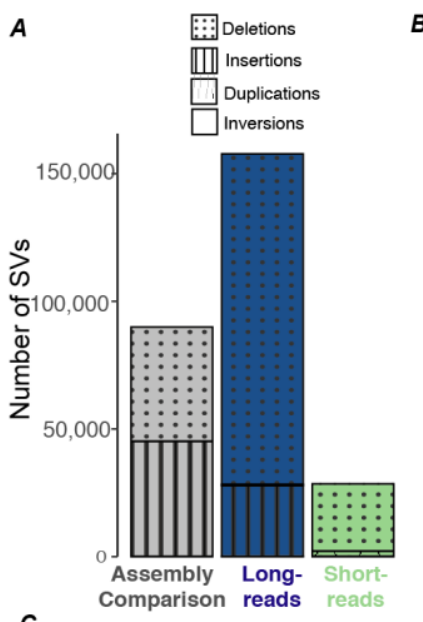

$B$

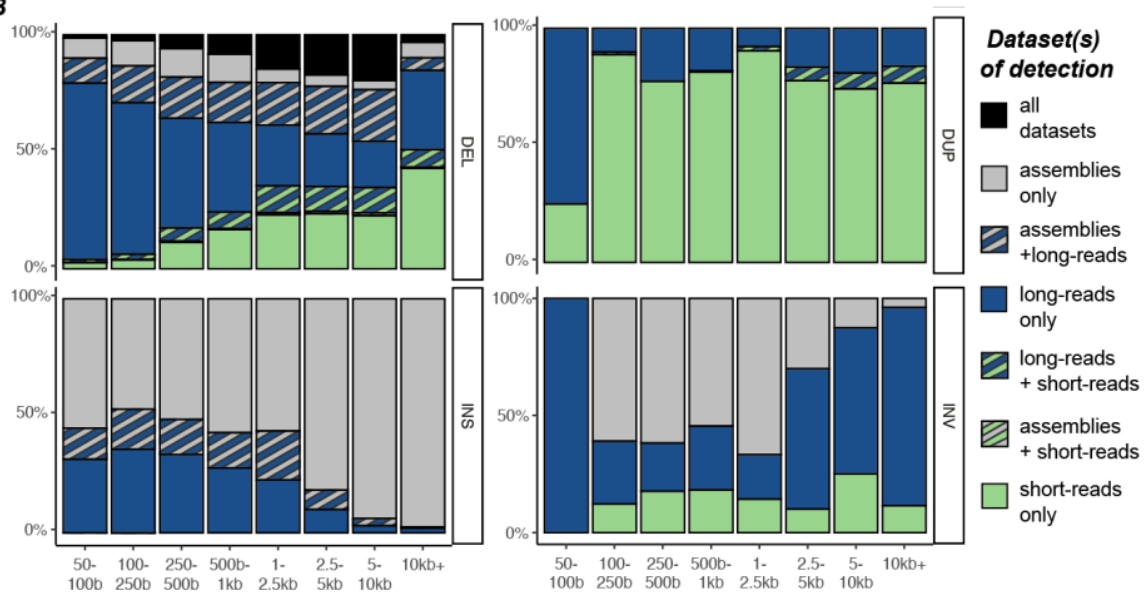

c

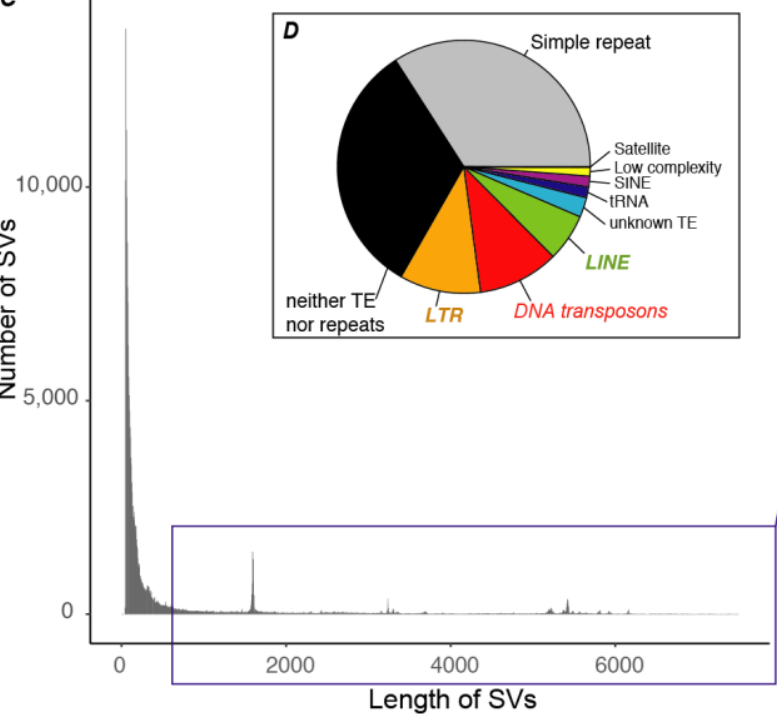

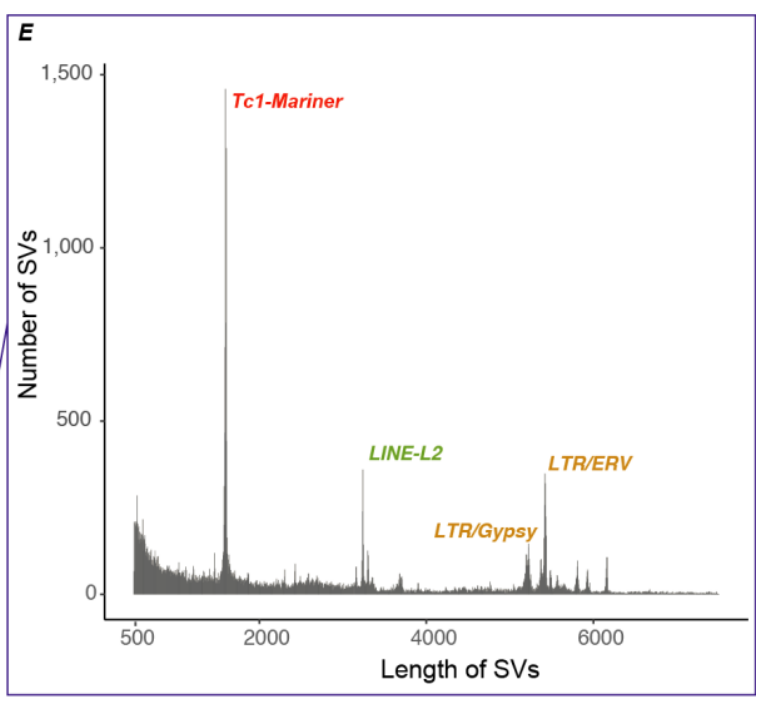

Figure 3: Overview of SVs detected in the Lake Whitefish

(A) Number of SVs detected in the three datasets by at least two tools, split by type (DEL/INS/DUP/INV). (B) Proportion of SVs detected in one or several datasets according to type and size. (C) Size distribution of SVs. (D) Proportion of SVs associated with different families of transposable elements and repeated elements (E) Size distribution of SVs (zoomed on the range 500-7500bp).

There was limited overlap between the different approaches with 7,525 SVs detected in the three datasets and 38,202 detected in two datasets out of a total of 222,927 SVs. Almost no overlap was observed for inversions and duplications, likely reflecting the difficulties in characterizing such SVs. For insertions, the overlap between long reads and assembly comparison approaches tended to decrease with size, possibly due to more approximative breakpoints, while for deletions it increased with size (Fig. 3B).

The distribution of SV sizes was highly-skewed towards smaller SVs below 500bp (Fig. 3C). We observed heterogeneous peaks in the SV size distribution corresponding to insertions or deletions of transposable elements (Fig. 3E). The sequence of SVs around the $1600 \mathrm{bp}$ peak matches with TC1- 
Mariner. SVs around $3700 \mathrm{bp}$ corresponds to Line-L2 indels while the peaks between 5000 and $6000 \mathrm{bp}$ are different kinds of LTR (Gypsy, ERV1). Overall, transposable elements were important factors driving structural variation in Lake Whitefish as their sequences were composed of $73 \%$ of transposable elements (compared to $60 \%$ for the entire genome, Table S4). This enrichment was mostly due to retroelements (49\% in SV sequences compared to $25 \%$ in the genome), mostly LTR and Gypsy (Table S5). This resulted in about a third of all SVs in the catalog being associated to an insertion or deletion of a TE (Fig. 3D). Satellite repeats and simple repeats (e.g. microsatellites) cover a smaller fraction of the SV sequences (5\%, Table S4) but they were found in about a third of SVs. A third of SVs did not match any TE nor any repeated regions.

\section{Polymorphism and differentiation in the Dwarf and Normal Lake Whitefish}

To assess genetic variation at the population level, we estimated genotype likelihoods for SNPs and SVs in the 32 samples sequenced with short reads. Filtering for genetic variants with allelic frequency higher than $5 \%$ retained $12,886,292$ SNPs and 103,857 SVs. Those "frequent" SVs cover a total of 66 $\mathrm{MB}$, representing polymorphism affecting approximately 5 times more nucleotides in the genome than SNPs.

Decomposing genetic variation with a principal component analysis displayed a strong clustering of individuals by species and by lake. This was consistent whether considering SNPs or SVs, although SVs tended to show greater separation between the two species along the $1^{\text {st }} P C$ (Fig. 4A-B). This suggests a higher level of shared inter-specific variation between lakes for SVs than for SNPs.

$\boldsymbol{A}$

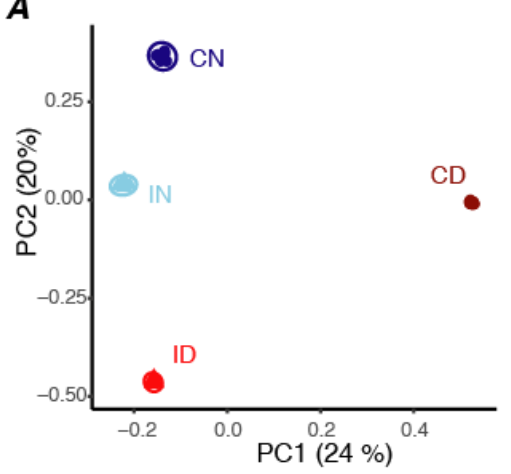

$B$

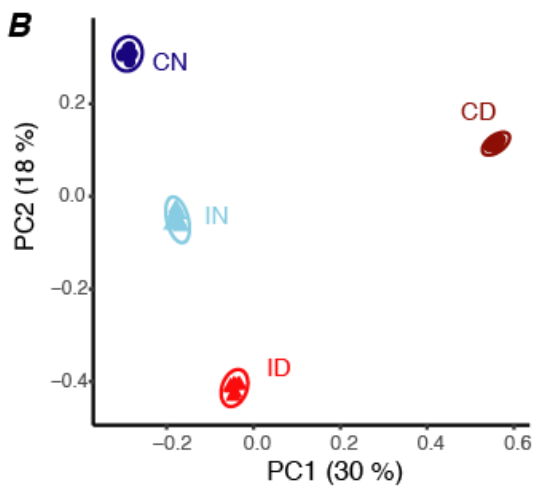

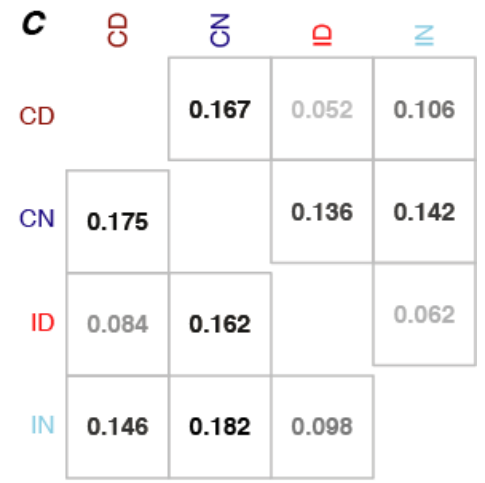

\section{Figure 4: Genomic variation in Lake Whitefish}

Principal component analysis (PCA) based on (A) SNPs and (B) SVs. Each point is an individual coloured by lake and by species. (C) FsT between lakes and species based on SNPs (below diagonal) and SVs (above diagonal). CN: Normal from Cliff Lake, CD: Dwarf from Cliff Lake, IN: Normal from Indian Lake, ID: Dwarf from Indian Lake

FST was moderate to high between lakes and between species, with values ranging from 0.052 up to 0.167 based on SVs and from 0.084 to 0.182 based on SNPs (Fig.4C-D). The Normal and Dwarf Whitefish were more differentiated in Cliff Lake than in Indian Lake using both kinds of variants (Cliff Lake: $\mathrm{F}_{\mathrm{ST}}=0.175 / 0.167$; Indian Lake: $\mathrm{F}_{\mathrm{ST}}=0.098 / 0.062$ ) and such species differentiation was widespread along the genome (Fig. 5). Within each lake, the landscape of interspecific F $_{S T}$ display similarities between SNPs and SVs, suggesting that there may be linked variants (for e.g. small deletions and SNPs) and that the two kinds of mutations may interact, for instance if some SVs capture SNPs and reduce recombination.

As the two lakes represent parallel situations of coexistence between the Normal and the Dwarf species of $C$. clupeaformis (Rougeux et al., 2017), we investigated whether genetic differentiation 
follows similar patterns. The most differentiated genetic variants, defined as the SNPs and SVs in the top $95 \%$ FST $_{\text {ST }}$ quantile within each lake, showed twice the expected number of shared variants across lakes, suggesting that areas of differentiation between species are conserved in parallel across lakes. When measuring species differentiation as a polarized difference in allelic frequencies (AFD statistic), this overlap was even stronger. There was a threefold excess for AFD outliers in the same end of the distribution (positive in both lakes and negative in both lakes) while there was a twofold deficit for AFD outliers in the opposite end of the distribution. In other words, the variants with high allelic frequency differences between species are more likely than expected by chance to display the same Normal allele and Dwarf allele in both lakes (Table 1).
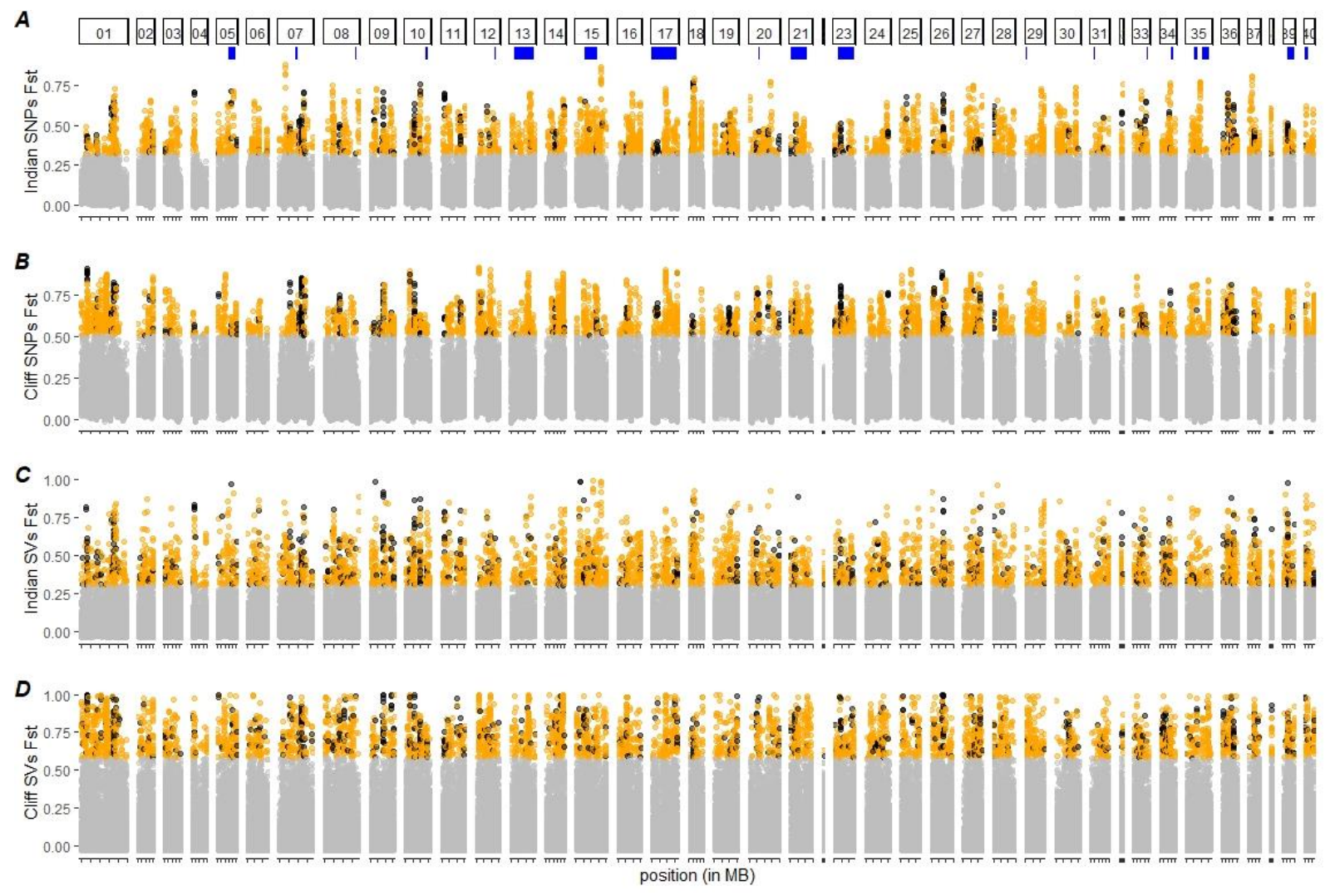

Figure 5: Genomic differentiation along the genome between Normal and Dwarf Whitefish

Fst between Normal and Dwarf Whitefish based on SNPs, by windows of 100kb, in (A) Indian Lake and (B) Cliff Lake. Fst between Normal and Dwarf species based on SVs in (C) Indian Lake and (D) Cliff Lake. Windows and variants that exceed the $95 \%$ quantile in one lake are colored in orange. Shared polymorphisms between lakes (i.e., variants found as outliers in both lakes) are shown in black. Blue segments under chromosome numbers indicate the positions of QTLs associated with behavioral and morphological differences between Normal and Dwarf species, as identified in Gagnaire et al (2013).

The most differentiated SVs, both within each lake and shared between lakes, were significantly enriched in TE-associated SVs. In contrast, SVs associated with simple repeats were under-represented in outliers of differentiation, while SVs without TE or repeats showed no bias. This excess of TE-linked SVs in outliers was driven by all categories of TE: DNA transposons, LINEs, SINEs and LTR. The most significantly enriched families in both lakes were the DNA transposons Tc1-mariner and hAT-Ac, and the retrotransposons LTR-Gypsy and LTR-ERV1, LINE-L1, LINE-L2 and LINE-RexBabar (Table 2, Table S6). 
Table 1: Overlap across the two lakes in the most differentiated variants between species.

AFD is the allelic frequency difference between the Dwarf and the Normal Whitefish (polarized as Dwarf-Normal). "Same sign" indicates that the outliers are in the same end of the AFD distribution in both lakes (either upper 97.5 quartile or lower 2.5 quartile), while "opposite sign" indicates that outliers are not in the same end of the AFD distribution in both lakes. In other words, outliers with opposite signs are variants in which the allele that is more frequent in the Dwarf in one lake is the allele that is more frequent in the Normal in the other lake.

\begin{tabular}{|c|c|c|c|c|c|}
\hline Dataset and method & $\begin{array}{c}\text { Number of } \\
\text { variants }\end{array}$ & $\begin{array}{c}\text { Expected } \\
\text { number of } \\
\text { overlapping } \\
\text { outliers at 5\% } \\
\text { limit }\end{array}$ & $\begin{array}{c}\text { Observed } \\
\text { number of } \\
\text { overlapping } \\
\text { outliers at } \\
5 \% \text { limit }\end{array}$ & $\begin{array}{l}\text { Odd- } \\
\text { ratio }\end{array}$ & $\begin{array}{c}\text { pvalue } \\
\text { (Fisher } \\
\text { test) }\end{array}$ \\
\hline SNP $F_{S T}$ & $9,215,577$ & 56,626 & 95,801 & 1.7 & $P<0.001$ \\
\hline SNP AFD same sign & $9,215,577$ & 28,812 & 81,386 & 2.8 & $P<0.001$ \\
\hline SNP AFD opposite sign & $9,215,577$ & 28,812 & 18,272 & 0.6 & $P<0.001$ \\
\hline$S V F_{S T}$ & 93,773 & 469 & 727 & 1.6 & $P<0.001$ \\
\hline SV AFD same sign & 93,773 & 234 & 618 & 2.6 & $P<0.001$ \\
\hline SV AFD opposite sign & 93,773 & 234 & 123 & 0.5 & $P<0.001$ \\
\hline
\end{tabular}

Table 2: Enrichment in SVs associated with transposable elements in outliers of differentiation between Dwarf and Normal Whitefish.

Population-scale analysis

(MAF>5\%)

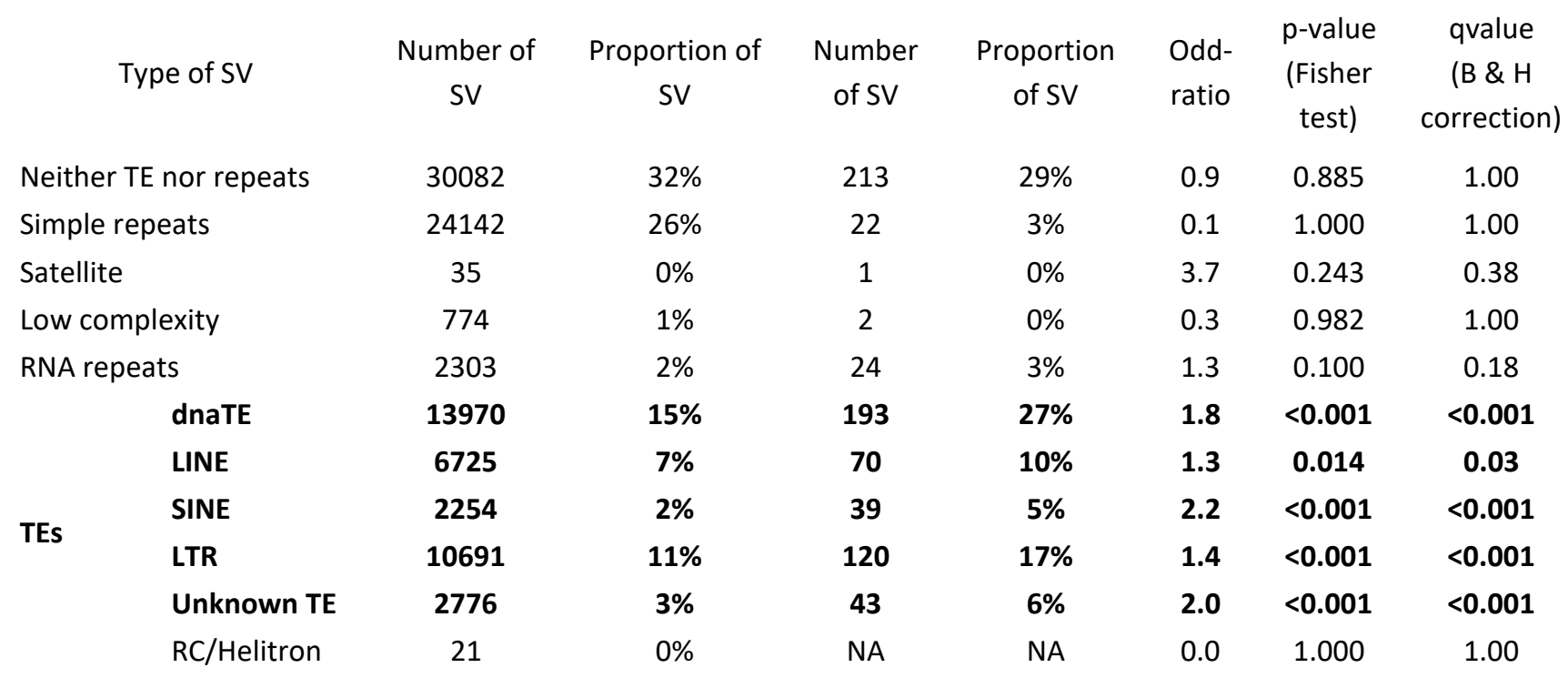

The most differentiated variants (SNPs and SVs) overlapped with thousands of genes. Gene ontology analysis revealed significant enrichment in behavior, morphogenesis, cell signaling, immunity and metabolism, among many others (Table S6). To narrow down putative candidate genes possibly involved in phenotypic differentiation, we focused on outliers overlapping with quantitative trait loci (QTL) previously mapped with the linkage map (Gagnaire, Normandeau, et al., 2013; Rogers et al., 
2007). A total of 27 QTLs for various traits differentiating Dwarf and Normal Whitefish (Growth rate, maturity, gill raker, etc) could be positioned on the new reference genome, although some of them had a relatively wide interval (Table S8, Fig 5). They overlapped with 45,823 SNPs and 414 SVs that were identified as outliers of differentiation in both lakes. The list of genes belonging to a QTL and overlapping with at least one outlier is provided in Table S9.

\section{Discussion:}

By combining long-read and short-read sequencing on Normal and Dwarf Whitefish, our study generated new genomic resources and provided insights into the genomic architecture of recent speciation. First, we have produced a reference genome assembly for both Normal and Dwarf Whitefish, as well as an extensive catalog of structural variants. Second, studying SVs at the populationlevel showed that SVs represent a large amount of variation within and between Normal and Dwarf Whitefish sympatric species, less numerous but more extensive than SNPs in terms of total number of nucleotides. Third, by comparing young species pairs in two lakes, we highlighted shared genetic differentiation and supported a predominant role of transposable elements in the recent divergence between the Normal and the Dwarf Whitefish. Hereafter, we discuss how our results and methods contribute to a better understanding of the genomic architecture of speciation and the involvement of structural variation.

Generating high-quality reference genomes for non-model species is becoming a requirement to understand the evolution of genomic variation during the speciation process (Nadeau \& Kawakami, 2019; Ravinet et al., 2017). Here, using a genome from the same species and the same geographic area as a reference facilitated the accurate detection of variants, both SNPs and SVs. Moreover, contiguity and chromosome-level information allowed a finer understanding of the role played by recombination, large rearrangements and chromosome-level variability (fusion/fission, karyotypic polymorphism, etc). In our study, the use of long-reads proved incomparable to resolve the complexity of the Dwarf and Normal Whitefish genomes. Using Nanopore sequencing data, we have been able to reach a high contiguity, allowing to search for structural variants. Assembly comparison, as well as direct detection based on long-reads, show that two individuals of Dwarf and Normal Whitefish differ by more than 100,000 high-confidence SVs. Given the stringency of our quality filters, and the lack of power to detect complex rearrangements or inversions, this number should be seen as a lower bound of the amount of SVs. In particular, most of the detected SVs remain in a range of small size $(<1 \mathrm{~kb})$ or relatively medium size. This catalog of SVs can therefore be supplemented by including more individuals, longer sequences, and additional genomes. In any case, the large number of high-confidence SVs identified in this study reinforces the importance of considering the possible role of SVs in speciation, which represents a novel proposed mechanism for speciation in the classical Whitefish species pairs system.

Regarding SVs of larger size ( $>100 \mathrm{~kb})$, we acknowledge that the detection power of our dataset was limited. Because the final scaffolding of the two genomes is based on a same (and not so dense) linkage map, made from a Normal x Dwarf hybrid family (Gagnaire, Normandeau, et al., 2013; Rogers et al., 2007), we could not search for large chromosomal rearrangements simply by contrasting the two genomes. This is unfortunate since large rearrangements such as inversions, fusions, and translocations may be relevant for speciation because they often differ between closely-related sympatric species and contribute to reproductive isolation (Berdan et al., 2021; Faria \& Navarro, 2010; Noor et al., 2001). In the case of the whitefish, on the one hand, we do not expect a major effect of chromosomal rearrangements. First, the differentiation observed in SNPs and SVs is widespread along 
the genome and does not display the typical spatial clustering of differentiated regions observed between species pairs like Littorina saxatilis (Morales et al., 2019) or Helianthus sp (Todesco et al., 2020). Second, cytogenetics analysis showed that the Dwarf and Normal Whitefish from these same lakes have an identical number of chromosomes (Dion-Côté et al., 2017). On the other hand, cytogenetic exploration showed subtle chromosomal polymorphism within and between them (DionCôté et al., 2017). For instance, chromosome 1 is longer in the Normal than in the Dwarf in Cliff Lake due to heterochromatin differences (Dion-Côté et al., 2017), a pattern that we also observed in the genome (121Mb vs. $99 \mathrm{Mb})$. We also note some peculiarities such as Chr22, for which the European sequences have homologous chromosomes in the American sequences but which we never managed to order as a full linkage group, likely because of the lack of recombination in the family used for the linkage map. Since the mother used for the linkage map is a hybrid Dwarf $x$ Normal, any rearrangement differing between species (and affecting recombination at heterozygote stage) may be absent from the final map, and hence from the present genomes. These chromosomal differences may lead to issues with recombination during meiosis (Dion-Côté et al., 2015; Faria \& Navarro, 2010), contributing to reproductive isolation and speciation (Hoffmann \& Rieseberg, 2008; Kirkpatrick \& Barton, 2006). In the future, it would be worthwhile to explore large scale chromosomal rearrangements in whitefish in depth to understand the role of chromosomal polymorphism in speciation. However, this will require improved genome scaffolding based on $\mathrm{Hi}-\mathrm{C}$ chromatin contacts (which was attempted here without success) or separate linkage maps.

Beyond the contrast between the Dwarf and Normal Whitefish species, the new genome assemblies also provide relevant information about the evolution of genomes at a higher taxonomic level. Salmonids have experienced a recent whole-genome duplication, followed by different events of rediploidization, as well as important chromosomal rearrangements such as fusions (Blumstein et al., 2020; Glasauer \& Neuhauss, 2014; Lien et al., 2016; Macqueen \& Johnston, 2014). Here, as often observed in salmonids, synteny was high between $C$. clupeaformis and closely related species such as the European whitefish Coregonus lavaretus "Balchen". The same groups of chromosomes appear to be metacentric and bear residual tetrasomy in $C$. clupeaformis as in its related species $C$. ardetii (Blumstein et al., 2020). Chromosomal comparison with C. ardetii and C. lavaretus also suggested shared fusion and fission of ancestral chromosomes and a consistent karyotype between the different coregonids (Blumstein et al., 2020; De-Kayne \& Feulner, 2018). This would suggest that the majority of re-deploidization processes occurred before the split of the different Coregonus species, which would all share a relatively similar karyotype. That being said, it should be kept in mind that the residual tetrasomy observed on a subset of chromosomes makes it difficult to fully ascertain synteny vs. rearrangements within and between species on those chromosomes. Moreover, $C$. clupeaformis genomes remain extremely complex with several regions that end up collapsed by genome assembly, as previously reported in other salmonid genomes (De-Kayne et al., 2020; Lien et al., 2016). Therefore, while the Coregonus reference genome assemblies provide a first important basis, refining the assemblies and complementing by cytogenetic or chromatin-contact data will be valuable to further explore of the timing and modalities of re-diploidization in coregonids, and its possible contribution to speciation.

Salmonids genomes are also littered with transposable elements and C. clupeaformis was no exception: interspersed repeats accounted for $60 \%$ of the genome. This amount is comparable to Salmo salar (60\% Lien et al. (2016)) and Coregonus lavaretus "Balchen" (52\% De-Kayne et al. (2020)). Moreover, all TE copies are not shared by all individuals and our results highlighted that they were 
responsible for a third of the SVs detected within and between species. This is also consistent with observations made on other species, such as Atlantic Salmon Salmo salar (Bertolotti et al., 2020) or songbird Corvus sp. (Weissensteiner et al., 2020), in which young and active TEs generate numerous insertions and deletions between samples. It has been hypothesized that bursts of transposon activity may contribute to speciation (de Boer, Yazawa, Davidson, \& Koop, 2007), or at least that TE may rapidly generate genetic variation differentiating species (Serrato-Capuchina \& Matute, 2018). Our data strongly support this hypothesis since the most differentiated SVs between Dwarf and Normal Whitefish in both lakes were enriched in several classes of TEs. A large part of the fixed genetic variation between species corresponds to an insertion or a deletion of a given TE. It is worth noting that this pattern is widespread across the genome rather than centered on a few loci. Such extensive differentiation suggests a progressive and differential TE accumulation without gene flow, likely in allopatry during the Pleistocene glaciation ( 15,000 generations/60,000 years ago) that may have contributed to the maintenance of reproductive isolation during the postglacial sympatric phase following secondary contact ( 3,000 generations/12,000 years ago) (Jacobsen et al., 2012; Rougeux et al., 2017). Accumulations of different TEs between lineages may be quite rapid as active TEs have a high mutation rate, as observed in Daphnia with an order or $10^{-5}$ gain or loss per copy per generation (Ho et al., 2021). TEs can also contribute to reproductive isolation by altering gene structure, expression pattern and chromosome organization (Dubin, Scheid, \& Becker, 2018; Goodier, 2016). Interestingly, TE de-regulation is known to generate post-zygotic breakdown in Dwarf $x$ Normal hybrids (Dion-Côté, Renaut, Normandeau, \& Bernatchez, 2014), which has been associated with epigenetic (methylation) reprogramming in hybrids (Laporte et al., 2019). Moreover, this supported the hypothesis that TE transcriptional de-repression, perhaps due to different TEs silencing mechanisms that evolved in allopatry, may be the cause for the previously documented abnormal embryonic development and death of hybrids (Renaut, Nolte, \& Bernatchez, 2009). Both in previous studies and in our study, the same TE families emerged as associated with species differentiation, namely Tc1mariner and hAT-Ac as well as LTR-Gypsy, Line-L2 and Line-RexBabar. Altogether then, cumulative evidence points towards a major role of several TE families in the reproductive isolation of Dwarf and Normal Whitefish, involving TE distributed throughout the genome rather than in a few barrier loci.

A peculiarity of Whitefish speciation is the independent, repeated evolution of two ecologically-distinct species (Dwarf and Normal) in separate lakes. Previous work revealed that strong parallelism at the phenotypic level was accompanied by weak parallelism at the genome level (Gagnaire, Pavey, et al., 2013; Rogers \& Bernatchez, 2007; Rougeux, Gagnaire, Praebel, Seehausen, \& Bernatchez, 2019). With a higher density of variants being screened, our results corroborate those from these previous studies. The pattern of differentiation between species was indeed specific to each lake. However, it is worth noting the excess of shared outliers of differentiation, both for SNPs and SVs, and that differences of allelic frequencies were more often in the same direction (e.g., higher allelic frequency in dwarf species in both lakes) than expected by chance. A large fraction of such parallelism likely reflects historical divergence between allopatric lineages, possibly reinforced by the result of comparable ecological response to selection. It is also possible that shared regions of differentiation reflect regions of the genome more resistant to gene flow, such as low recombination regions, as observed in Ficedula flycatchers (Burri et al., 2015). General patterns of TE enrichment in outlier SVs, as well as gene ontology enrichment, also converged between lakes. This suggests that the processes driving genetic divergence between species were likely similar between lakes, namely through shared historical divergence and similar ecological selection imposed by the use of distinct trophic niches 
(Bernatchez et al., 2010), although they were buffered by lake-specific contingency at finer molecular level, for instance associated with the effect of genetic drift on available standing genetic variation within each lake (Gagnaire, Pavey, et al., 2013).

Studying two types of genetic variants in tandem, SVs and SNPs, at the population level showed similar patterns and level of differentiation between species and between lakes. On the one hand, this confirms that evaluating population/species structure requires neither a diversity of variants nor a large amount of markers. In fact, the FST values observed at the scale of the entire genome for both types of variants and in both lakes were strikingly similar to values measured based on a much smaller subset of markers. For instance, based on the RADseq genotyping of about 2500 SNP loci, Gagnaire,

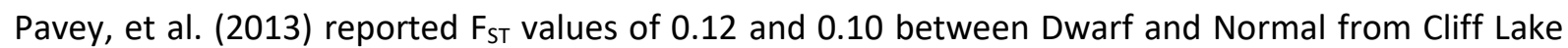
and from Indian Lake respectively, compared to values of 0.18 and 0.10 here for SNPs and 0.17 and 0.06 for SVs. On the other hand, studying different kinds of variants with similar filters shows a large amount of non-rare SVs, i.e. SVs found in more than 2 of 64 chromosomes. Because of their size, the accumulation of SVs at intermediate frequency in natural populations thus represents a non-negligible aspect of genetic variation, as they covered at least five times more of the genome than SNPs. This point is increasingly underlined by studies in population genomics and evolutionary genomics (Catanach et al., 2019; Mérot et al., 2020; Weissensteiner et al., 2020) and means that a full understanding of genetic variation cannot overlook SVs. However, it remains difficult to study SVs at the population level. Short reads are more accessible when sequencing a large number of individuals but they proved to be less powerful for characterizing SVs (Mahmoud et al., 2019). For instance, here we found around 5 times fewer SVs with 32 samples sequenced with short-reads than with 2 samples sequenced with long reads. Recent studies propose to rely on mixed datasets (e.g. combining longread and short-read sequence data) to achieve together a good catalog of SV and then perform population genomic studies based on their variation (Logsdon et al., 2020). We have achieved this in this study by first characterizing SVs using high-quality long reads on a limited number of samples, and secondly by genotyping known SVs with short-reads on a greater number of samples. To achieve this, genome-graph based approaches were particularly relevant, allowing us to build a variation-aware reference graph (Garrison et al., 2018), and then perform unbiased mapping of reads to this graph (Sirén et al., 2020). Such two-step approaches have also be used in a handful of studies looking at SVs in chocolate trees Theobroma cacao (Hämälä et al., 2021), soybeans Glycine max (Lemay et al., 2021), and potato beetle Leptinotarsa decemlineata (Cohen, Hawthorne, \& Schoville, 2021). Based on this, we believe that the combination of $2^{\text {nd }}$ and $3^{\text {rd }}$ generation sequencing is promising to study structural variation within a population genomics framework and will allow the inclusion of SVs in studies of speciation and adaptation genomics.

\section{Acknowledgements:}

We would like to thank M. Suga for contributing to fieldwork, K. Wellband for help with annotation, M-A. Lemay for help with SV analysis, M. Leitwein for help with the analysis of metacentric chromosomes, A-L Ferchaud and R. De Kayne for their help with synteny analysis and plotting. We are also very grateful to the teams that developed $\mathrm{vg}$, giraffe and pggb for their guidance in using the genome-graph tools as well as J. Laroche at the IBIS Bioinformatic platform (www.ibis.ulaval.ca) for his support. 


\section{Funding:}

This research was supported by a Discovery research grant from the Natural Sciences and Engineering Research Council of Canada (NSERC) to L.B., the Canadian Research Chair in genomics and conservation of aquatic resources, as well as Ressources Aquatiques Québec (RAQ). C.M. was supported by a Banting Postdoctoral fellowship.

\section{Data Availability:}

Final genome assemblies are available on NCBI under the id ASM1839867v1 and ASM2061545v1. Longreas sequences and short-read sequences will be available on NCBI under project number XXX upon publication. Code are available on Github for the different pipelines listed in the methods.

\section{Author contributions:}

This study is part of the long-term research program of L.B. on the adaptive radiation and ecological speciation of Whitefish. L.B., C.R. and C.M. conceived the study. M.Á. and M.K. performed the Nanopore sequencing. M.M and S.L. assembled the genome and E.N. and C.M. scaffolded the genome. J.M.F. analysed the transposable elements. C.M. and K.S. did the analyses with contributions from C.V., E.N., M.L. and S.L. C.M. wrote the manuscript with contributions from all authors.

\section{References:}

Abel, H. J., Larson, D. E., Regier, A. A., Chiang, C., Das, I., Kanchi, K. L., ... Reeves, C. (2020). Mapping and characterization of structural variation in 17,795 human genomes. Nature, 583(7814), 83-89.

Aljanabi, S. M., \& Martinez, I. (1997). Universal and rapid salt-extraction of high quality genomic DNA for PCR-based techniques. Nucleic Acids Research, 25(22), 4692-4693.

Allendorf, F. W., Bassham, S., Cresko, W. A., Limborg, M. T., Seeb, L. W., \& Seeb, J. E. (2015). Effects of crossovers between homeologs on inheritance and population genomics in polyploidderived salmonid fishes. Journal of Heredity, 106(3), 217-227.

Allendorf, F. W., \& Thorgaard, G. H. (1984). Tetraploidy and the evolution of salmonid fishes. In Evolutionary genetics of fishes (pp. 1-53). Springer.

Alonge, M., Soyk, S., Ramakrishnan, S., Wang, X., Goodwin, S., Sedlazeck, F. J., ... Schatz, M. C. (2019). RaGOO: fast and accurate reference-guided scaffolding of draft genomes. Genome Biology, 20(1), 1-17.

Berdan, E. L., Fuller, R. C., \& Kozak, G. M. (2021). Genomic landscape of reproductive isolation in Lucania killifish: The role of sex loci and salinity. Journal of Evolutionary Biology, 34(1), 157174.

Bernatchez, L., Chouinard, A., \& Lu, G. (1999). Integrating molecular genetics and ecology in studies of adaptive radiation: Whitefish, Coregonus sp., as a case study. Biological Journal of the Linnean Society, 68(1-2), 173-194.

Bernatchez, L., \& Dodson, J. J. (1990). Allopatric origin of sympatric populations of lake whitefish (Coregonus clupeaformis) as revealed by mitochondrial-DNA restriction analysis. Evolution, 44(5), 1263-1271.

Bernatchez, L., Renaut, S., Whiteley, A. R., Derome, N., Jeukens, J., Landry, L., ... Rogers, S. M. (2010). On the origin of species: Insights from the ecological genomics of lake whitefish. Philosophical Transactions of the Royal Society B: Biological Sciences, 365(1547), 1783-1800. 
Bertolotti, A. C., Layer, R. M., Gundappa, M. K., Gallagher, M. D., Pehlivanoglu, E., Nome, T., ... Macqueen, D. J. (2020). The structural variation landscape in 492 Atlantic salmon genomes. Nature Communications, 11(1), 5176. doi: 10.1038/s41467-020-18972-x

Blumstein, D. M., Campbell, M. A., Hale, M. C., Sutherland, B. J., McKinney, G. J., Stott, W., \& Larson, W. A. (2020). Comparative genomic analyses and a novel linkage map for cisco (Coregonus artedi) provide insights into chromosomal evolution and rediploidization across salmonids. G3: Genes, Genomes, Genetics, 10(8), 2863-2878.

Breese, M. R., \& Liu, Y. (2013). NGSUtils: A software suite for analyzing and manipulating nextgeneration sequencing datasets. Bioinformatics, 29(4), 494-496.

Burri, R., Nater, A., Kawakami, T., Mugal, C. F., Olason, P. I., Smeds, L., ... Garamszegi, L. Z. (2015). Linked selection and recombination rate variation drive the evolution of the genomic landscape of differentiation across the speciation continuum of Ficedula flycatchers. Genome Research, 25(11), 1656-1665.

Cabanettes, F., \& Klopp, C. (2018). D-GENIES: dot plot large genomes in an interactive, efficient and simple way. PeerJ, 6, e4958.

Catanach, A., Crowhurst, R., Deng, C., David, C., Bernatchez, L., \& Wellenreuther, M. (2019). The genomic pool of standing structural variation outnumbers single nucleotide polymorphism by threefold in the marine teleost Chrysophrys auratus. Molecular Ecology, 28(6), 1210-1223.

Catchen, J., Amores, A., \& Bassham, S. (2020). Chromonomer: A tool set for repairing and enhancing assembled genomes through integration of genetic maps and conserved synteny. BioRxiv.

Chaisson, M. J., Sanders, A. D., Zhao, X., Malhotra, A., Porubsky, D., Rausch, T., ... Collins, R. L. (2019). Multi-platform discovery of haplotype-resolved structural variation in human genomes. Nature Communications, 10(1), 1-16.

Chakraborty, M., Baldwin-Brown, J. G., Long, A. D., \& Emerson, J. (2016). Contiguous and accurate de novo assembly of metazoan genomes with modest long read coverage. Nucleic Acids Research, 44(19), e147-e147.

Chen, S., Zhou, Y., Chen, Y., \& Gu, J. (2018). fastp: An ultra-fast all-in-one FASTQ preprocessor. Bioinformatics, 34(17), i884-i890.

Chen, X., Schulz-Trieglaff, O., Shaw, R., Barnes, B., Schlesinger, F., Källberg, M., ... Saunders, C. T. (2016). Manta: Rapid detection of structural variants and indels for germline and cancer sequencing applications. Bioinformatics, 32(8), 1220-1222.

Cohen, Z., Hawthorne, D., \& Schoville, S. (2021). The role of structural variants in pest adaptation and genome evolution of the Colorado potato beetle, Leptinotarsa decemlineata (Say). Authorea Preprints.

Crete-Lafreniere, A., Weir, L. K., \& Bernatchez, L. (2012). Framing the Salmonidae family phylogenetic portrait: A more complete picture from increased taxon sampling.

Cruickshank, T. E., \& Hahn, M. W. (2014). Reanalysis suggests that genomic islands of speciation are due to reduced diversity, not reduced gene flow. Molecular Ecology, 23(13), 3133-3157.

Dalziel, A. C., Laporte, M., Rougeux, C., Guderley, H., \& Bernatchez, L. (2017). Convergence in organ size but not energy metabolism enzyme activities among wild Lake Whitefish (Coregonus clupeaformis) species pairs. Molecular Ecology, 26(1), 225-244.

de Boer, J. G., Yazawa, R., Davidson, W. S., \& Koop, B. F. (2007). Bursts and horizontal evolution of DNA transposons in the speciation of pseudotetraploid salmonids. BMC Genomics, 8(1), 110. 
De-Kayne, R., \& Feulner, P. G. (2018). A European whitefish linkage map and its implications for understanding genome-wide synteny between salmonids following whole genome duplication. G3: Genes, Genomes, Genetics, 8(12), 3745-3755.

De-Kayne, R., Zoller, S., \& Feulner, P. G. (2020). A de novo chromosome-level genome assembly of Coregonus sp."Balchen": One representative of the Swiss Alpine whitefish radiation. Molecular Ecology Resources, 20(4), 1093-1109.

Dion-Côté, A., Symonová, R., Lamaze, F. C., Pelikánová, Š., Ráb, P., \& Bernatchez, L. (2017). Standing chromosomal variation in Lake Whitefish species pairs: The role of historical contingency and relevance for speciation. Molecular Ecology, 26(1), 178-192.

Dion-Côté, A.-M., Renaut, S., Normandeau, E., \& Bernatchez, L. (2014). RNA-seq reveals transcriptomic shock involving transposable elements reactivation in hybrids of young lake whitefish species. Molecular Biology and Evolution, 31(5), 1188-1199.

Dion-Côté, A.-M., Symonová, R., Ráb, P., \& Bernatchez, L. (2015). Reproductive isolation in a nascent species pair is associated with aneuploidy in hybrid offspring. Proceedings of the Royal Society B: Biological Sciences, 282(1802), 20142862.

Dubin, M. J., Scheid, O. M., \& Becker, C. (2018). Transposons: A blessing curse. Current Opinion in Plant Biology, 42, 23-29.

Dufresnes, C., Brelsford, A., Jeffries, D. L., Mazepa, G., Suchan, T., Canestrelli, D., ... Crochet, P.-A. (2021). Mass of genes rather than master genes underlie the genomic architecture of amphibian speciation. Proceedings of the National Academy of Sciences, 118(36), e2103963118. doi: 10.1073/pnas.2103963118

Evans, M., \& Bernatchez, L. (2012). Oxidative phosphorylation gene transcription in whitefish species pairs reveals patterns of parallel and nonparallel physiological divergence. Journal of Evolutionary Biology, 25(9), 1823-1834.

Faria, R., \& Navarro, A. (2010). Chromosomal speciation revisited: Rearranging theory with pieces of evidence. Trends in Ecology \& Evolution, 25(11), 660-669.

Feulner, P., Chain, F. J., Panchal, M., Eizaguirre, C., Kalbe, M., Lenz, T. L., ... Milinski, M. (2013). Genome-wide patterns of standing genetic variation in a marine population of three-spined sticklebacks. Molecular Ecology, 22(3), 635-649.

Feulner, P., \& De-Kayne, R. (2017). Genome evolution, structural rearrangements and speciation. Journal of Evolutionary Biology, 30(8), 1488-1490.

Flynn, J. M., Hubley, R., Goubert, C., Rosen, J., Clark, A. G., Feschotte, C., \& Smit, A. F. (2020). RepeatModeler2 for automated genomic discovery of transposable element families. Proceedings of the National Academy of Sciences, 117(17), 9451-9457.

Gagnaire, P., Normandeau, E., Pavey, S. A., \& Bernatchez, L. (2013). Mapping phenotypic, expression and transmission ratio distortion QTL using RAD markers in the Lake Whitefish (Coregonus clupeaformis). Molecular Ecology, 22(11), 3036-3048.

Gagnaire, P., Pavey, S. A., Normandeau, E., \& Bernatchez, L. (2013). The genetic architecture of reproductive isolation during speciation-with-gene-flow in lake whitefish species pairs assessed by RAD sequencing. Evolution, 67(9), 2483-2497.

Garrison, E., Sirén, J., Novak, A. M., Hickey, G., Eizenga, J. M., Dawson, E. T., ... Lin, M. F. (2018). Variation graph toolkit improves read mapping by representing genetic variation in the reference. Nature Biotechnology, 36(9), 875-879.

Glasauer, S. M., \& Neuhauss, S. C. (2014). Whole-genome duplication in teleost fishes and its evolutionary consequences. Molecular Genetics and Genomics, 289(6), 1045-1060. 
Goel, M., Sun, H., Jiao, W.-B., \& Schneeberger, K. (2019). SyRI: finding genomic rearrangements and local sequence differences from whole-genome assemblies. Genome Biology, 20(1), 1-13.

Goodier, J. L. (2016). Restricting retrotransposons: A review. Mobile DNA, 7(1), 1-30.

Gu, Z., Gu, L., Eils, R., Schlesner, M., \& Brors, B. (2014). Circlize implements and enhances circular visualization in R. Bioinformatics, 30(19), 2811-2812.

Hämälä, T., Wafula, E. K., Guiltinan, M. J., Ralph, P. E., dePamphilis, C. W., \& Tiffin, P. (2021). Genomic structural variants constrain and facilitate adaptation in natural populations of Theobroma cacao, the chocolate tree. Proceedings of the National Academy of Sciences, 118(35).

Harris, R. S. (2007). Improved pairwise alignment of genomic DNA. The Pennsylvania State University.

Hejase, H. A., Salman-Minkov, A., Campagna, L., Hubisz, M. J., Lovette, I. J., Gronau, I., \& Siepel, A. (2020). Genomic islands of differentiation in a rapid avian radiation have been driven by recent selective sweeps. Proceedings of the National Academy of Sciences, 117(48), 30554. doi: 10.1073/pnas.2015987117

Heller, D., \& Vingron, M. (2019). SVIM: structural variant identification using mapped long reads. Bioinformatics, 35(17), 2907-2915.

Heller, D., \& Vingron, M. (2020). SVIM-asm: Structural variant detection from haploid and diploid genome assemblies. Bioinformatics, 36(22-23), 5519-5521.

Henderson, E. C., \& Brelsford, A. (2020). Genomic differentiation across the speciation continuum in three hummingbird species pairs. BMC Evolutionary Biology, 20(1), 1-11.

Hickey, G., Heller, D., Monlong, J., Sibbesen, J. A., Sirén, J., Eizenga, J., ... Paten, B. (2020). Genotyping structural variants in pangenome graphs using the vg toolkit. Genome Biology, 21(1), 1-17.

Ho, E. K. H., Bellis, E. S., Calkins, J., Adrion, J. R., Latta IV, L. C., \& Schaack, S. (2021). Engines of change: Transposable element mutation rates are high and variable within Daphnia magna. PLOS Genetics, 17(11), e1009827. doi: 10.1371/journal.pgen.1009827

Ho, Urban, A. E., \& Mills, R. E. (2019). Structural variation in the sequencing era. Nature Reviews Genetics, 21(3), 171-189.

Hoffmann, A. A., \& Rieseberg, L. H. (2008). Revisiting the impact of inversions in evolution: From population genetic markers to drivers of adaptive shifts and speciation? Annual Review of Ecology, Evolution, and Systematics, 39, 21-42.

Huddleston, J., Ranade, S., Malig, M., Antonacci, F., Chaisson, M., Hon, L., ... Dennis, M. Y. (2014). Reconstructing complex regions of genomes using long-read sequencing technology. Genome Research, gr-168450.

Jacobsen, M. W., Hansen, M. M., Orlando, L., Bekkevold, D., Bernatchez, L., Willerslev, E., \& Gilbert, M. T. P. (2012). Mitogenome sequencing reveals shallow evolutionary histories and recent divergence time between morphologically and ecologically distinct European whitefish (Coregonus spp.). Molecular Ecology, 21(11), 2727-2742.

Jain, C., Rhie, A., Zhang, H., Chu, C., Walenz, B. P., Koren, S., \& Phillippy, A. M. (2020). Weighted minimizer sampling improves long read mapping. Bioinformatics, 36(Supplement_1), i111i118.

Jiggins, C. D. (2019). Can genomics shed light on the origin of species? PLOS Biology, 17(8), e3000394. doi: 10.1371/journal.pbio.3000394

Kirkpatrick, M., \& Barton, N. (2006). Chromosome inversions, local adaptation and speciation. Genetics, 173(1), 419-434.

Kirsche, M., Prabhu, G., Sherman, R., Ni, B., Aganezov, S., \& Schatz, M. C. (2021). Jasmine: Population-scale structural variant comparison and analysis. BioRxiv. 
Kolmogorov, M., Yuan, J., Lin, Y., \& Pevzner, P. A. (2019). Assembly of long, error-prone reads using repeat graphs. Nature Biotechnology, 37(5), 540-546.

Korneliussen, T. S., Albrechtsen, A., \& Nielsen, R. (2014). ANGSD: analysis of next generation sequencing data. BMC Bioinformatics, 15(1), 356.

Landis, J. B., Soltis, D. E., Li, Z., Marx, H. E., Barker, M. S., Tank, D. C., \& Soltis, P. S. (2018). Impact of whole-genome duplication events on diversification rates in angiosperms. American Journal of Botany, 105(3), 348-363.

Landry, L., Vincent, W., \& Bernatchez, L. (2007). Parallel evolution of lake whitefish dwarf ecotypes in association with limnological features of their adaptive landscape. Journal of Evolutionary Biology, 20(3), 971-984.

Laporte, M, Le Luyer, J., Rougeux, C., Dion-Côté, A.-M., Krick, M., \& Bernatchez, L. (2019). DNA methylation reprogramming, TE derepression, and postzygotic isolation of nascent animal species. Science Advances, 5(10), eaaw1644.

Laporte, Martin, Dalziel, A. C., Martin, N., \& Bernatchez, L. (2016). Adaptation and acclimation of traits associated with swimming capacity in Lake Whitefish (Coregonus clupeaformis) ecotypes. BMC Evolutionary Biology, 16(1), 1-13.

Laporte, Martin, Rogers, S. M., Dion-Côté, A.-M., Normandeau, E., Gagnaire, P.-A., Dalziel, A. C., ... Bernatchez, L. (2015). RAD-QTL mapping reveals both genome-level parallelism and different genetic architecture underlying the evolution of body shape in lake whitefish (Coregonus clupeaformis) species pairs. G3: Genes, Genomes, Genetics, 5(7), 1481-1491.

Layer, R. M., Chiang, C., Quinlan, A. R., \& Hall, I. M. (2014). LUMPY: a probabilistic framework for structural variant discovery. Genome Biology, 15(6), R84.

Legendre, P., \& Legendre, L. F. (2012). Numerical ecology (Vol. 24). Elsevier.

Lemay, M.-A., Sibbesen, J. A., Torkamaneh, D., Hamel, J., Levesque, R. C., \& Belzile, F. (2021). Combined use of Oxford Nanopore and Illumina sequencing yields insights into soybean structural variation biology. BioRxiv.

Li, H. (2018). Minimap2: Pairwise alignment for nucleotide sequences. Bioinformatics, 34(18), 30943100 .

Li, H., \& Durbin, R. (2009). Fast and accurate short read alignment with Burrows-Wheeler transform. Bioinformatics, 25(14), 1754-1760.

Li, H., Feng, X., \& Chu, C. (2020). The design and construction of reference pangenome graphs with minigraph. Genome Biology, 21(1), 1-19.

Li, H., Handsaker, B., Wysoker, A., Fennell, T., Ruan, J., Homer, N., ... Durbin, R. (2009). The sequence alignment/map format and SAMtools. Bioinformatics, 25(16), 2078-2079.

Lien, S., Koop, B. F., Sandve, S. R., Miller, J. R., Kent, M. P., Nome, T., ... Zimin, A. (2016). The Atlantic salmon genome provides insights into rediploidization. Nature, 533(7602), 200-205.

Limborg, M. T., McKinney, G. J., Seeb, L. W., \& Seeb, J. E. (2016). Recombination patterns reveal information about centromere location on linkage maps. Molecular Ecology Resources, 16(3), 655-661.

Logsdon, G. A., Vollger, M. R., \& Eichler, E. E. (2020). Long-read human genome sequencing and its applications. Nature Reviews Genetics, 21(10), 597-614.

Lou, R. N., Jacobs, A., Wilder, A., \& Therkildsen, N. O. (2020). A beginner's guide to low-coverage whole genome sequencing for population genomics. 
Macqueen, D. J., \& Johnston, I. A. (2014). A well-constrained estimate for the timing of the salmonid whole genome duplication reveals major decoupling from species diversification. Proceedings of the Royal Society B: Biological Sciences, 281(1778), 20132881.

Mahmoud, M., Gobet, N., Cruz-Dávalos, D. I., Mounier, N., Dessimoz, C., \& Sedlazeck, F. J. (2019). Structural variant calling: The long and the short of it. Genome Biology, 20(1), 246.

Marçais, G., Delcher, A. L., Phillippy, A. M., Coston, R., Salzberg, S. L., \& Zimin, A. (2018). MUMmer4: A fast and versatile genome alignment system. PLoS Computational Biology, 14(1), e1005944.

Marques, D. A., Lucek, K., Meier, J. I., Mwaiko, S., Wagner, C. E., Excoffier, L., \& Seehausen, O. (2016). Genomics of rapid incipient speciation in sympatric threespine stickleback. PLoS Genetics, 12(2), e1005887.

Martin, S. H., Davey, J. W., Salazar, C., \& Jiggins, C. D. (2019). Recombination rate variation shapes barriers to introgression across butterfly genomes. PLoS Biology, 17(2), e2006288.

McKenna, A., Hanna, M., Banks, E., Sivachenko, A., Cibulskis, K., Kernytsky, A., ... Daly, M. (2010). The Genome Analysis Toolkit: A MapReduce framework for analyzing next-generation DNA sequencing data. Genome Research, 20(9), 1297-1303.

Meier, J. I., Marques, D. A., Wagner, C. E., Excoffier, L., \& Seehausen, O. (2018). Genomics of parallel ecological speciation in Lake Victoria cichlids. Molecular Biology and Evolution, 35(6), 14891506.

Meisner, J., \& Albrechtsen, A. (2018). Inferring population structure and admixture proportions in low-depth NGS data. Genetics, 210(2), 719-731.

Mérot, C., Oomen, R. A., Tigano, A., \& Wellenreuther, M. (2020). A roadmap for understanding the evolutionary significance of structural genomic variation. Trends in Ecology \& Evolution, 35(7), 561-572.

Morales, H. E., Faria, R., Johannesson, K., Larsson, T., Panova, M., Westram, A. M., \& Butlin, R. K. (2019). Genomic architecture of parallel ecological divergence: Beyond a single environmental contrast. Science Advances, 5(12), eaav9963.

Nadeau, N. J., \& Kawakami, T. (2019). Population Genomics of Speciation and Admixture. In O. P. Rajora (Ed.), Population Genomics: Concepts, Approaches and Applications (pp. 613-653). Cham: Springer International Publishing. doi: 10.1007/13836_2018_24

Noor, M. A., Grams, K. L., Bertucci, L. A., Almendarez, Y., Reiland, J., \& Smith, K. R. (2001). The genetics of reproductive isolation and the potential for gene exchange between Drosophila pseudoobscura and D. persimilis via backcross hybrid males. Evolution, 55(3), 512-521.

Ou, S., \& Jiang, N. (2019). LTR_FINDER_parallel: Parallelization of LTR_FINDER enabling rapid identification of long terminal repeat retrotransposons. Mobile DNA, 10(1), 1-3.

Phillips, R. B., Reed, K. M., \& Ráb, P. (1996). Revised karyotypes and chromosome banding of coregonid fishes from the Laurentian Great Lakes. Canadian Journal of Zoology, 74(2), 323329.

Rastas, P. (2017). Lep-MAP3: Robust linkage mapping even for low-coverage whole genome sequencing data. Bioinformatics, 33(23), 3726-3732. doi: 10.1093/bioinformatics/btx494

Rausch, T., Zichner, T., Schlattl, A., Stütz, A. M., Benes, V., \& Korbel, J. O. (2012). DELLY: structural variant discovery by integrated paired-end and split-read analysis. Bioinformatics, 28(18), i333-i339.

Ravinet, M., Faria, R., Butlin, R., Galindo, J., Bierne, N., Rafajlović, M., ... Westram, A. (2017). Interpreting the genomic landscape of speciation: A road map for finding barriers to gene flow. Journal of Evolutionary Biology, 30(8), 1450-1477. 
Renaut, S., Nolte, A., \& Bernatchez, L. (2009). Transcriptomic investigation of post-zygotic isolation in lake whitefish (Coregonus clupeaformis). Mol. Biol. Evol, 26, 925-936.

Rogers, \& Bernatchez, L. (2007). The genetic architecture of ecological speciation and the association with signatures of selection in natural lake whitefish (Coregonus sp. Salmonidae) species pairs. Molecular Biology and Evolution, 24(6), 1423-1438.

Rogers, S. M., Gagnon, V., \& Bernatchez, L. (2002). Genetically based phenotype-environment association for swimming behavior in lake whitefish ecotypes (Coregonus clupeaformis Mitchill). Evolution, 56(11), 2322-2329.

Rogers, S. M., Isabel, N., \& Bernatchez, L. (2007). Linkage maps of the dwarf and normal lake whitefish (Coregonus clupeaformis) species complex and their hybrids reveal the genetic architecture of population divergence. Genetics, 175(1), 375-398.

Rougeux, Bernatchez, L., \& Gagnaire, P.-A. (2017). Modeling the multiple facets of speciation-withgene-flow toward inferring the divergence history of lake whitefish species pairs (Coregonus clupeaformis). Genome Biology and Evolution, 9(8), 2057-2074.

Rougeux, Gagnaire, P., Praebel, K., Seehausen, O., \& Bernatchez, L. (2019). Polygenic selection drives the evolution of convergent transcriptomic landscapes across continents within a Nearctic sister species complex. Molecular Ecology, 28(19), 4388-4403.

Scannell, D. R., Byrne, K. P., Gordon, J. L., Wong, S., \& Wolfe, K. H. (2006). Multiple rounds of speciation associated with reciprocal gene loss in polyploid yeasts. Nature, 440(7082), 341345.

Sedlazeck, F. J., Rescheneder, P., Smolka, M., Fang, H., Nattestad, M., Von Haeseler, A., \& Schatz, M. C. (2018). Accurate detection of complex structural variations using single-molecule sequencing. Nature Methods, 15(6), 461-468.

Seehausen, O., Butlin, R. K., Keller, I., Wagner, C. E., Boughman, J. W., Hohenlohe, P. A., ... Brännström, Å. (2014). Genomics and the origin of species. Nature Reviews Genetics, 15(3), 176-192.

Serrato-Capuchina, A., \& Matute, D. R. (2018). The role of transposable elements in speciation. Genes, 9(5), 254.

Sirén, J., Monlong, J., Chang, X., Novak, A. M., Eizenga, J. M., Markello, C., ... Carroll, A. (2020). Genotyping common, large structural variations in 5,202 genomes using pangenomes, the Giraffe mapper, and the vg toolkit. BioRxiv.

Soderlund, C., Bomhoff, M., \& Nelson, W. M. (2011). SyMAP v3. 4: A turnkey synteny system with application to plant genomes. Nucleic Acids Research, 39(10), e68-e68.

Stevison, L. S., \& McGaugh, S. E. (2020). It's time to stop sweeping recombination rate under the genome scan rug. Molecular Ecology, 29(22), 4249-4253. doi: 10.1111/mec.15690

Sutherland, B. J. G., Gosselin, T., Normandeau, E., Lamothe, M., Isabel, N., Audet, C., \& Bernatchez, L. (2016). Salmonid Chromosome Evolution as Revealed by a Novel Method for Comparing RADseq Linkage Maps. Genome Biology and Evolution, 8(12), 3600-3617. doi: 10.1093/gbe/evw262

Tham, C. Y., Tirado-Magallanes, R., Goh, Y., Fullwood, M. J., Koh, B. T., Wang, W., ... Tenen, D. G. (2020). NanoVar: Accurate characterization of patients' genomic structural variants using low-depth nanopore sequencing. Genome Biology, 21(1), 1-15.

Ting, C.-T., Tsaur, S.-C., Sun, S., Browne, W. E., Chen, Y.-C., Patel, N. H., \& Wu, C.-I. (2004). Gene duplication and speciation in Drosophila: Evidence from the Odysseus locus. Proceedings of the National Academy of Sciences, 101(33), 12232-12235. 
Todesco, M., Owens, G. L., Bercovich, N., Légaré, J.-S., Soudi, S., Burge, D. O., ... Imerovski, I. (2020). Massive haplotypes underlie ecotypic differentiation in sunflowers. Nature, 584(7822), 602607.

Trudel, M., Tremblay, A., Schetagne, R., \& Rasmussen, J. B. (2001). Why are dwarf fish so small? An energetic analysis of polymorphism in lake whitefish (Coregonus clupeaformis). Canadian Journal of Fisheries and Aquatic Sciences, 58(2), 394-405.

Walker, B. J., Abeel, T., Shea, T., Priest, M., Abouelliel, A., Sakthikumar, S., ... Young, S. K. (2014). Pilon: An integrated tool for comprehensive microbial variant detection and genome assembly improvement. PloS One, 9(11), e112963.

Waples, R. K., Seeb, L. W., \& Seeb, J. E. (2016). Linkage mapping with paralogs exposes regions of residual tetrasomic inheritance in chum salmon (Oncorhynchus keta). Molecular Ecology Resources, 16(1), 17-28.

Weissensteiner, M. H., Bunikis, I., Catalán, A., Francoijs, K.-J., Knief, U., Heim, W., ... Suh, A. (2020). Discovery and population genomics of structural variation in a songbird genus. Nature Communications, 11(1), 1-11.

Wellenreuther, M., \& Bernatchez, L. (2018). Eco-evolutionary genomics of chromosomal inversions. Trends in Ecology \& Evolution, 33(6), 427-440.

Wolf, J. B., \& Ellegren, H. (2017). Making sense of genomic islands of differentiation in light of speciation. Nature Reviews Genetics, 18(2), 87-100. 